\title{
Transient strain distributions during femtosecond laser-induced deformation inside LiF and MgO single crystals
}

\section{$\operatorname{AUTHOR}(\mathrm{S}):$}

Sakakura, Masaaki; Shimotsuma, Yasuhiko; Fukuda, Naoaki; Miura, Kiyotaka

\section{CITATION:}

Sakakura, Masaaki ... [et al]. Transient strain distributions during femtosecond laserinduced deformation inside LiF and MgO single crystals. Journal of Applied Physics 2015, 118(2): 023106.

\section{ISSUE DATE:}

2015-07-14

URL:

http://hdl.handle.net/2433/201606

\section{RIGHT:}

(C) 2015 American Institute of Physics. This article may be downloaded for personal use only. Any other use requires prior permission of the author and the American Institute of Physics. 


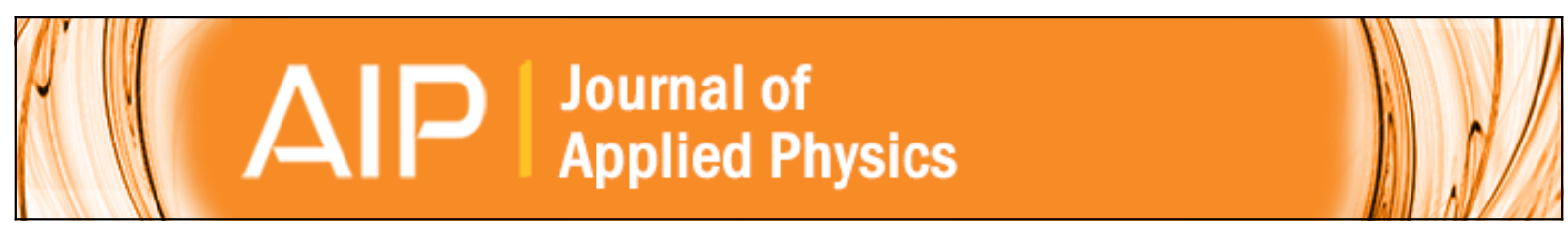

Transient strain distributions during femtosecond laser-induced deformation inside LiF and $\mathrm{MgO}$ single crystals

Masaaki Sakakura, Yasuhiko Shimotsuma, Naoaki Fukuda, and Kiyotaka Miura

Citation: Journal of Applied Physics 118, 023106 (2015); doi: 10.1063/1.4926614

View online: http://dx.doi.org/10.1063/1.4926614

View Table of Contents: http://scitation.aip.org/content/aip/journal/jap/118/2?ver=pdfcov

Published by the AIP Publishing

\section{Articles you may be interested in}

Mechanism of heat-modification inside a glass after irradiation with high-repetition rate femtosecond laser pulses J. Appl. Phys. 108, 073533 (2010); 10.1063/1.3483238

Anisotropic lattice changes in femtosecond laser inscribed $\mathrm{Nd} 3+: \mathrm{MgO}: \mathrm{LiNbO} 3$ optical waveguides J. Appl. Phys. 106, 013110 (2009); 10.1063/1.3168432

Femtosecond real-time probing of transition state dynamics in a surface photoreaction: Methyl desorption from $\mathrm{CH} 3 \mathrm{I}$ on $\mathrm{MgO}(100)$

J. Chem. Phys. 129, 011105 (2008); 10.1063/1.2953578

Enhanced $1.5 \mu$ m emission and simultaneously suppressed green upconversion emission in Er : Li Nb O 3 crystals heavily codoped with $\mathrm{MgO}$

Appl. Phys. Lett. 91, 071914 (2007); 10.1063/1.2769749

Optical investigation of femtosecond laser induced microstress in neodymium doped lithium niobate crystals J. Appl. Phys. 100, 033521 (2006); 10.1063/1.2218991

\section{AIP $\left.\right|_{\text {APL Photonics }}$}

APL Photonics is pleased to announce Benjamin Eggleton as its Editor-in-Chief

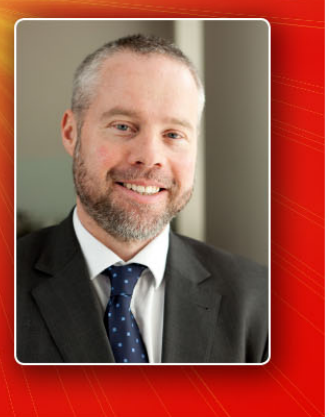




\title{
Transient strain distributions during femtosecond laser-induced deformation inside LiF and MgO single crystals
}

\author{
Masaaki Sakakura, ${ }^{1, a)}$ Yasuhiko Shimotsuma, ${ }^{2}$ Naoaki Fukuda, ${ }^{1}$ and Kiyotaka Miura ${ }^{2}$ \\ ${ }^{1}$ Hitachi Zosen Collaborative Research Division, Office of Society-Academia Collaboration for Innovation, \\ Kyoto University, Goryo-ohara 1-30, Kyoto 615-8245, Japan \\ ${ }^{2}$ Department of Material Chemistry, Graduate School of Engineering, Kyoto University, \\ Kyoutodaigaku-katsura, Kyoto 615-8510, Japan
}

(Received 6 May 2015; accepted 30 June 2015; published online 14 July 2015)

\begin{abstract}
Strain and stress dynamics inside $\mathrm{MgO}$ and $\mathrm{LiF}$ single crystals after photoexcitation by a focused femtosecond laser pulse were investigated by the observation of transient distributions of birefringence around the photoexcited region using a time-resolved polarization microscope. Both in $\mathrm{MgO}$ and $\mathrm{LiF}$, propagation of two stress waves, which were attributed to quasi-longitudinal and quasi-transverse elastic waves, were observed, but crack propagation was observed only in LiF. Inside $\mathrm{MgO}$, the observed strain distributions could be reproduced by elastic simulation, whereas inside $\mathrm{LiF}$ the strain distributions during crack propagation were largely different from the simulated ones; strain was widely distributed between cracks and the $\langle 110\rangle$ regions in a stress wave, the strained region around the photoexcited region was smaller, and the strains in the $\langle 110\rangle$ region and near the crack tips were larger than those by the simulation. The amplitudes of strain and stress in stress waves and temperature change in the photoexcited region were evaluated, and the origins of strain distribution change due to crack generation were discussed based on the differences between $\mathrm{MgO}$ and LiF. (C) 2015 AIP Publishing LLC. [http://dx.doi.org/10.1063/1.4926614]
\end{abstract}

\section{INTRODUCTION}

In crystalline solids, characteristic deformations occur under stress or shock loading. ${ }^{1-8}$ For example, in rock-salt crystals, such as sodium chloride $(\mathrm{NaCl})$, magnesium oxide $(\mathrm{MgO})$, and lithium fluoride $(\mathrm{LiF})$, slips and generation of dislocations tend to occur in the $\langle 110\rangle$ directions under a shear stress, and cleavage cracks are formed normal to $\{100\}$ planes under a tensile stress or shock. ${ }^{1,2}$ On the other hand, in non-crystalline solids such as glasses, deformation is often accompanied with cracks in random directions. ${ }^{9}$ Because the cleavage cracks can be used as rapid crystal cutting and dislocations alter the local material strength, understanding the tendency of these deformations by stresses has been one of the important issues to develop a precise material processing technique.

Generation of cracks and dislocations by stress or shock have been observed also in pulsed-laser processing, because stress or shock wave is generated as a result of ablation on the surface or fast thermal expansion inside materials. ${ }^{10-16}$ In the femtosecond (fs) laser bulk processing inside transparent solid materials [Fig. 1(a)], a stress wave is generated from the photoexcited region by nonlinear ionization at the laser focal region, and cracks and dislocations are generated in the specific directions inside $\mathrm{MgO}, \mathrm{LiF}$, and $\mathrm{Al}_{2} \mathrm{O}_{3}$ single crystals. ${ }^{5-7}$ For example, inside a $\mathrm{MgO}$ single crystal, of which crystal system is a rock-salt, birefringent regions of dislocations (dislocation band) appear in the $\langle 110\rangle$ directions from the photoexcited region after focusing a fs laser pulse normal to the (001) surface [Figs. 1(b) and 1(d)].

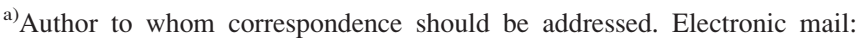
msakakura@saci.kyoto-u.ac.jp
}

Dislocation bands appear in the $\langle 110\rangle$ directions also inside a LiF single crystal, but cracks are formed parallel to the $\{100\}$ planes at the same photoexcitation energy as that in $\mathrm{MgO}$ [Figs. 1(c) and 1(d)]. ${ }^{6}$ Such characteristic deformations inside single crystals are often explained in terms of their crystal systems and bond energies. ${ }^{1}$ However, the mechanism of these deformations cannot be explained only by their crystal systems, because stress loading is necessary for inducing these deformations. Therefore, for understanding the mechanism of the fs laser-induced deformations inside transparent materials, it is essential to elucidate the stress dynamics after photoexcitation. If the relation between the stress dynamics and deformations is elucidated, we will be able to understand the mechanism of the stress or shock induced deformations of solids as well as find methods to control deformation by controlled stresses.

We have investigated fs laser-induced deformation and dynamics inside a $\mathrm{MgO}$ and $\mathrm{LiF}$ single crystals. Because these crystals have a simple structure (cubic) and no birefringence, ${ }^{1}$ they are suitable for understanding the basic mechanism of laser-induced deformations as model dielectric materials. In addition, investigation of laser-induced deformation in $\mathrm{MgO}$ and $\mathrm{LiF}$ is also important for optical applications, because they have wide transmission windows in infrared, visible, and ultraviolet regions. ${ }^{17}$ In our previous study, we observed a laser-induced stress wave of characteristic shape inside $\mathrm{MgO}$ and $\mathrm{LiF}$ single crystals by a pumpprobe transmission microscope, and speculated that the shape of the stress wave should be the origins of cracks and dislocations; in the stress waves, the shear stress and tensile stress were largest in the $\langle 110\rangle$ and $\langle 100\rangle$ directions, respectively, and they were attributed to the driving forces of the 
(a)

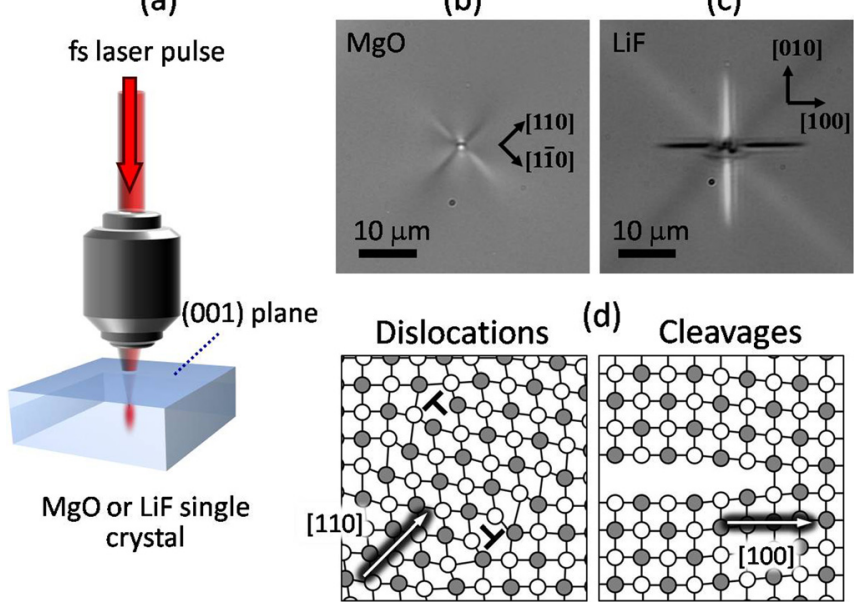

FIG. 1. (a) Schematic illustration of photoexcitation inside a $\mathrm{MgO}$ or $\mathrm{LiF}$ single crystal by focusing fs laser pulses. (b) and (c) Polarization microscope images of fs laser-induced modifications inside a $\mathrm{MgO}$ and $\mathrm{LiF}$ single crystal, respectively. (d) Schematic illustrations of two main deformations inside $\mathrm{MgO}$ and $\mathrm{LiF}$ single crystals.

generation of the dislocations and cracks. ${ }^{6}$ However, in the study, we could not elucidate the amplitudes or directions of the strain and stress that induce these structural changes. In addition, the difference in stress dynamics with and without crack generation, i.e., between $\mathrm{LiF}$ and $\mathrm{MgO}$, has not been elucidated clearly. Elucidation of the difference in the stress dynamics between $\mathrm{LiF}$ and $\mathrm{MgO}$ is important to understand the mechanism of crack generation, because the difference could come from the crack generation.

Stress and strains inside transparent materials can be visualized through strain-induced birefringence, which has been known as a photoelastic effect. ${ }^{18}$ Because birefringence is related to the amplitude and direction of strain and can be analyzed by the polarization change of a probe light, ${ }^{18,19}$ it is possible to visualize transient strain distributions after photoexcitation inside transparent materials by polarization detection. In this study, to clarify the strain and stress distributions and the difference in strain dynamics between $\mathrm{MgO}$ and $\mathrm{LiF}$, we developed a pump-probe polarization microscope $^{20}$ and observed transient distributions of birefringence inside $\mathrm{MgO}$ and LiF single crystals after photoexcitation by a focused fs laser pulse. We found that the observed strain dynamics could be reproduced by the elastic simulation in $\mathrm{MgO}$, in which cracks were not generated, whereas the observed strain dynamics were quite different from the simulated dynamics in $\mathrm{LiF}$, in which cracks were generated. Based on the observation, we evaluated the amplitudes of strain and stress by laser induced stress wave and temperature increase in the photoexcitation, and discussed the mechanism of dislocation and crack generation and the origins of differences in the strain dynamics between $\mathrm{MgO}$ and $\mathrm{LiF}$.

\section{EXPERIMENTAL AND SIMULATION}

\section{A. Pump-probe polarization microscope}

A schematic illustration of the pump-probe polarization microscope is shown in Fig. 2(a). A 120 fs laser pulse of $800 \mathrm{~nm}$ wavelength (mode-locked Ti-sapphire laser with a regenerative amplifier, Mira-Legend; Coherent Inc.) was divided into two by a beam splitter. One of the split pulses was used as a pump pulse, and the other was passed through a $\mathrm{BBO}\left(\beta-\mathrm{BaB}_{2} \mathrm{O}_{4}\right)$ crystal to generate the second harmonic (wavelength: $400 \mathrm{~nm}$ ) and used as a probe pulse. The pump pulse was focused inside a $\mathrm{MgO}$ or $\mathrm{LiF}$ single crystal $\left(10 \times 10 \times 1.0 \mathrm{~mm}^{3}\right)$ with a $50 \times$ objective lens $($ Nikon, LU Plan $50 \times$, N.A. $=0.8)$ to induce nonlinear-photoexcitation inside the crystal. The incident of the pump pulse was normal to the (001) surface of the crystal and the depth of the focus was about $0.1 \mathrm{~mm}$ from the surface of the sample. Because the photoexcited volume depends on the depth of the focus due to a spherical aberration, the depth of the focus was the same through the experiment. The probe pulse was
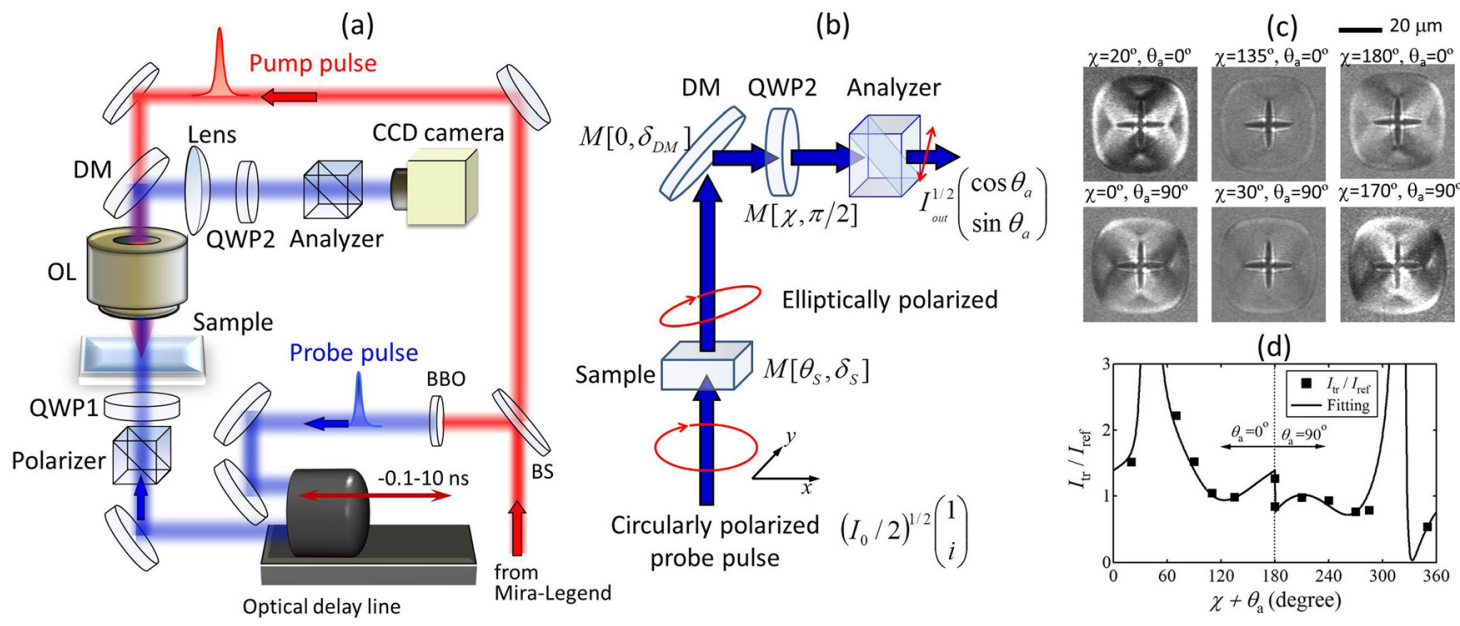

FIG. 2. (a) Optical setup of a pump-probe polarization microscope. BS: a beam splitter, QWP1, QWP2: quarter waveplates, DM: dichroic mirror, OL: 50× objective lens, Lens: a convex lens of $f=300 \mathrm{~mm}$. The analyzer was removed in observation of transmission images. (b) Schematic illustration of the principle for the measurement of birefringence by the optical setup of (a). The probe pulse before the crystal is circularly polarized and the intensity is $I_{0}$. The probe light just before detection is linearly polarized and the intensity is $I_{\text {out }}$. (c) Ratios of transient images and reference images obtained under different $\chi$ and $\theta_{\mathrm{a}}$ by the pump-probe measurement of (a). These were the images at $3 \mathrm{~ns}$ after irradiation with a focused fs laser pulse of $2 \mu \mathrm{J}$ inside a LiF single crystal. (d) Plot of $I_{\mathrm{tr}} /$ $I_{\text {ref }}$ at a single pixel in the images of (c) against $\chi+\theta_{\mathrm{a}}$ and fitting with Eq. (3). 
optically delayed by an optical delay line and converted to a circularly polarized light by a polarizer and quarter waveplate (QWP1). The zero-delay was determined by monitoring the optical Kerr effect by the pump pulse (the detail is described in the supplementary material ${ }^{21}$ ). The probe pulse was transmitted through the photoexcited region inside the crystal from the opposite direction of the pump pulse. The transmitted probe pulse was magnified by the objective lens, and the transmission image around the photoexcited region was focused on a charge coupled device (CCD) camera by a convex lens $(f=300 \mathrm{~mm})$. Before being detected by the $\mathrm{CCD}$, the probe pulse was reflected by a dichroic mirror (DM) and transmitted through a quarter waveplate (QWP2) and analyzer.

A probe image by a single photoexcitation was obtained by a single image acquisition by the CCD camera, and four images were averaged to obtain the intensity distribution of the probe pulse. In a single data acquisition, two probe images, before and after photoexcitation, were obtained. We call probe images before and after photoexcitation "reference image" and "transient image," respectively. Every single data acquisition, the crystal was moved by $100 \mu \mathrm{m}$ perpendicular to the optical axis of the pump pulse to avoid the influence by the previous photoexcitation. For analysis of transient birefringence, the intensity distributions of the probe pulse were obtained at various orientations of the QWP2 $(\chi)$ and analyzer $\left(\theta_{\mathrm{a}}\right)$, and the birefringence distributions were obtained by analyzing the relation between the intensity distribution and $\chi$. The method for the analysis is explained in Subsection II B.

\section{B. Analysis of birefringence}

The analysis of the birefringence is based on the calculation of polarization change of the probe beam in the pumpprobe polarization microscope. For the analysis, the polarization change was calculated by the Jones calculus. ${ }^{22}$ The polarization change of the probe pulse is illustrated schematically in Fig. 2(b). Because the probe pulse before transmitting trough the crystal is circularly polarized, the probe pulse before the sample is expressed by $\left(I_{0} / 2\right)^{1 / 2}(1, i)^{\tau}$, where $I_{0}$ and $\tau$ denote the intensity of the probe pulse and transposition for the vector, respectively. The polarization of the probe pulse is altered at three stages; at transmission through the sample, reflection on the DM and transmission through the QWP2. When the angle of QWP2 is $\chi$, the retardance by the DM is $\delta_{\mathrm{DM}}$, the azimuth and retardance of the birefringence of the sample are $\theta_{S}$, and $\delta_{S}$, respectively, and the angle of the analyzer is $\theta_{\mathrm{a}}$, the light intensity after the analyzer $\left(I_{\text {out }}\right)$ can be expressed by

$$
\begin{aligned}
I_{\text {out }}\left(\chi ; \theta_{a} ; \theta_{S}, \delta_{S}\right)= & \frac{1}{2} I_{0} \mid\left(\begin{array}{cc}
\cos ^{2} \theta_{a} & \sin \theta_{a} \cos \theta_{a} \\
\sin \theta_{a} \cos \theta_{a} & \sin ^{2} \theta_{a}
\end{array}\right) \\
& \times\left. M(\chi, \pi / 2) M\left(0^{\circ}, \delta_{D M}\right) M\left(\theta_{S}, \delta_{S}\right)\left(\begin{array}{l}
1 \\
i
\end{array}\right)\right|^{2},
\end{aligned}
$$

where $M(\alpha, \delta)$ is the Jones Matrix which is defined by

$$
M(\alpha, \delta)=\left(\begin{array}{cc}
\cos \alpha & \sin \alpha \\
-\sin \alpha & \cos \alpha
\end{array}\right)\left(\begin{array}{cc}
1 & 0 \\
0 & e^{i \delta}
\end{array}\right)\left(\begin{array}{cc}
\cos \alpha & -\sin \alpha \\
\sin \alpha & \cos \alpha
\end{array}\right),
$$

where $\alpha$ and $\delta$ are the azimuth of the slow-axis and retardance of the birefringence. For example, $\delta$ of a quarter waveplate is $\pi / 2$, which corresponds to a quarter of a wavelength. In this experiment, the DM was aligned so that the angle of the birefringence was parallel to the $\mathrm{x}$ axis of this system $\left(\alpha_{\mathrm{DM}}=0^{\circ}\right)$. The retardance by the DM, $\delta_{\mathrm{DM}}$, was determined as $\delta_{\mathrm{DM}}=-0.078 \pi$ independently.

The intensity of a transient image $\left(I_{\text {tr }}\right)$ is given by $I_{\mathrm{tr}}=I_{\text {out }}\left(\chi, \theta_{\mathrm{a}} ; \theta_{S}, \delta_{S}\right)$, because birefringence appears in a crystal by photoexcitation. The intensity of a reference image $\left(I_{\text {rer }}\right)$ is given by $I_{\text {out }}\left(\chi, \theta_{\mathrm{a}} ; 0^{\circ}, 0\right)$, because there is no birefringence in a crystal before photoexcitation. To cancel out $I_{0}$, we calculated the ratio between the intensities of the intensities of transient and reference images, $I_{\mathrm{tr}} / I_{\mathrm{ref}}=R\left(\chi, \theta_{\mathrm{a}}\right.$; $\left.\theta_{S}, \delta_{S}\right) . R\left(\chi, \theta_{\mathrm{a}} ; \theta_{S}, \delta_{S}\right)$ can be expressed by

$$
R\left(\chi, \theta_{a} ; \theta_{S}, \delta_{S}\right)=I_{\text {out }}\left(\chi, \theta_{a} ; \theta_{S}, \delta_{S}\right) / I_{\text {out }}\left(\chi, \theta_{a} ; 0^{\circ}, 0\right) .
$$

In the experiment, the reference image and transient image were obtained as a function of $\chi$ and $\theta_{\mathrm{a}}=0^{\circ}$ or $90^{\circ}$, and the ratio between the transient image $\left[I_{\mathrm{tr}}\left(\chi, \theta_{a}\right)\right]$ and reference image $\left[I_{\text {ref }}\left(\chi, \theta_{a}\right)\right]$ was calculated as shown in Fig. 2(c). To obtain the birefringence at one pixel on a $\operatorname{CCD}, I_{\mathrm{tr}}\left(\chi, \theta_{a}\right) / I_{\mathrm{ref}}\left(\chi, \theta_{a}\right)$ was fitted by nonlinear least squared method with a fitting function of Eq. (3). The fitting parameters are $\theta_{S}$ and $\delta_{S}$. The example of a plot of $I_{\text {tr }}\left(\chi, \theta_{a}\right) / I_{\text {ref }}\left(\chi, \theta_{a}\right)$ against $\chi+\theta_{\mathrm{a}}$ and fitting with Eq. (3) are shown in Fig. 2(d).

\section{Simulation of a fs laser-induced strain dynamics}

The transient strain distributions inside $\mathrm{MgO}$ and $\mathrm{LiF}$ single crystals were simulated based on the elastic dynamics. ${ }^{23}$ We assumed that the main origin of a fs laser-induced stress wave was a thermal stress in the photoexcited region and the thermal stress was generated instantaneously after the photoexcitation. The distribution of the temperature change in the photoexcited region $[\Delta T(x, y, z)]$ was expressed by a Gaussian function as follows:

$$
\Delta T(x, y, z)=\Delta T_{\max } * \exp \left[-\frac{x^{2}+y^{2}}{\left(w_{t h} / 2\right)^{2}}-\frac{z^{2}}{\left(l_{z} / 2\right)^{2}}\right],
$$

where $\Delta T_{\max }$ is the temperature change at the peak due to photoexcitation, $x, y$, and $\mathrm{z}$ are the coordinates in the material, $w_{\text {th }}$ and $l_{\mathrm{z}}$ are, respectively, the width and length of the heated region. Here, $\mathrm{z}$ is the propagation direction of a fs laser pulse. In this study, a fs laser pulse was focused normal to the (001) plane of a $\mathrm{MgO}$ and $\mathrm{LiF}$ single crystal, and we defined $\mathrm{x}, \mathrm{y}$, and $\mathrm{z}$ as [100], [010], and [001] of the cubic system, respectively. According to the elastic dynamics, the temporal evolution of displacements in a material can be calculated by the following equation of motion:

$$
\rho \frac{\partial^{2} \mathbf{u}(x, y, z ; t)}{\partial t^{2}}=\nabla \cdot\left[\mathbf{P}(x, y, z ; t)-\mathbf{P}^{(\text {thermal })}(x, y, z)\right],
$$

where $t$ is the time after the photoexcitation, $\rho$ is the density of the material, $\boldsymbol{u}(x, y, z ; t)$ is the displacement vector at $t$, $\boldsymbol{P}(x, y, z ; t)$ is the stress tensor due to the displacement, and 
$\boldsymbol{P}^{\text {(thermal) }}(x, y, z)$ is the thermal stress tensor due to the temperature change. According to the Hook's law, the components of $\boldsymbol{P}(x, y, z ; t)$ in a crystal of cubic system can be expressed by

$$
P_{i i}=C_{11} S_{i i}+C_{23}\left(S_{j j}+S_{k k}\right) \quad(i, j, k=x, y, z ; i \neq j \neq k \neq i),
$$

$$
P_{i j}=2 C_{44} S_{i j} \quad(i \neq j),
$$

where $P_{\mathrm{ij}}$ and $S_{\mathrm{ij}}$ are the components of the stress and strain tensor, respectively, and $C_{\mathrm{ij}}$ are the components in the elastic tensor of the material. The strain tensor, $S(x, y, z ; t)$, was calculated by

$$
\mathbf{S}(x, y, z ; t)=\frac{1}{2}\left[\nabla \mathbf{u}+(\nabla \mathbf{u})^{\tau}\right] .
$$

The thermal stress tensor in a crystal of cubic system can be expressed by

$$
\mathbf{P}_{i j}^{(\text {thermal })}(x, y, z)=\beta \Delta T(x, y, z)\left(C_{11}+2 C\right) \delta_{i j},
$$

where $\beta$ is the thermal expansion coefficient of the material and $\delta_{\mathrm{ij}}$ is the Kronecker delta. Equation (5) with Eqs. (4) and (6)-(9) were calculated numerically to obtain the distribution of the displacement in a crystal $[\boldsymbol{u}(x, y, z ; t)]$ after the generation of thermal stress by photoexcitation. The densities, thermal expansion coefficients, and elastic constants of $\mathrm{MgO}$ and $\mathrm{LiF}$ single crystals for the numerical calculation were listed in Table I. ${ }^{24-27}$

\section{Calculation of strain and stress}

Strain and stress distributions were calculated by an observed distribution of birefringence, of which retardance and azimuth are, respectively, $\delta_{\mathrm{S}}$ and $\theta_{\mathrm{S}}$, using the photoelastic constants of a material. The relations between strain and strain-induced birefringence in a cubic system are given by $^{18,19}$

$$
\begin{gathered}
\left|S_{x x}-S_{y y}\right|=\delta_{S} \frac{\lambda_{\text {probe }}}{n_{0}^{3} \pi l}\left|\frac{\cos 2 \theta_{S}}{p_{11}-p_{12}}\right|, \\
2\left|S_{x y}\right|=\delta_{S} \frac{\lambda_{\text {probe }}}{n_{0}^{3} \pi l}\left|\frac{\sin 2 \theta_{S}}{2 p_{44}}\right|
\end{gathered}
$$

where $S_{\mathrm{xx}}, S_{\mathrm{yy}}$, and $S_{\mathrm{xy}}$ are the strain tensor components, $n_{0}$ is the refractive index of the material, $p_{11}, p_{12}$, and $p_{44}$ are the photoelastic constants of the material, $\lambda_{\text {probe }}(=400 \mathrm{~nm}$ in this study) is the wavelength of a probe pulse, and $l$ is the length of the birefringent region in the probe beam axis.

TABLE I. Properties of $\mathrm{MgO}$ and $\mathrm{LiF}$ single crystals used in this study. Densities $\left(\rho / \mathrm{gcm}^{-3}\right),{ }^{24,25}$ thermal expansion coefficient $\left(\beta / \mathrm{K}^{-1}\right),{ }^{26,27}$ elastic constants $\left(\mathrm{C}_{\mathrm{ij}} / \mathrm{GPa}\right),{ }^{24,25}$ refractive indices $\left(\mathrm{n}_{0}\right),{ }^{29,30}$ photoelastic constants $\left(\mathrm{p}_{\mathrm{ij}}\right),{ }^{29,31}$ and surface energies $\left(\gamma_{\mathrm{B}} / \mathrm{Jm}^{-2}\right)$ of $\mathrm{MgO}$ and LiF single crystals. ${ }^{32}$

\begin{tabular}{lccccccccc}
\hline \hline & $\rho$ & $\beta$ & $\mathrm{C}_{11}$ & $\mathrm{C}_{23}$ & $\mathrm{C}_{44}$ & $\mathrm{n}_{0}$ & $\mathrm{p}_{11-} \mathrm{p}_{12}$ & $\mathrm{p}_{44}$ & $\gamma_{\mathrm{B}}$ \\
\hline $\mathrm{MgO}$ & 3.58 & $1.1 \times 10^{-5}$ & 297 & 95 & 156 & 1.7 & -0.25 & -0.10 & 1.2 \\
$\mathrm{LiF}$ & 2.64 & $3.3 \times 10^{-5}$ & 111 & 42 & 63 & 1.4 & -0.10 & -0.043 & 0.34 \\
\hline \hline
\end{tabular}

Here, we assumed that the strains in the $\mathrm{z}$ direction (parallel to the propagation of a pump pulse) are negligible. Using Eqs. (10) and (11), the principal strain can be expressed by

$$
\left|S_{r}-S_{\theta}\right|=\delta_{S} \frac{\lambda_{\text {probe }}}{n_{0}^{3} \pi l}\left|\left(\frac{\cos 2 \theta_{S}}{p_{11}-p_{12}}\right)^{2}+\left(\frac{\sin 2 \theta_{S}}{2 p_{44}}\right)^{2}\right|^{0.5},
$$

where $S_{\mathrm{r}}$ and $S_{\theta}$ are the principal strains. In an optically isotropic material, $\left|p_{11}-p_{12}\right|$ is equal to $\left|2 p_{44}\right|$ (for example, in a silica glass, $\mathrm{p}_{11}-\mathrm{p}_{12}=-0.145$ and $\left.\mathrm{p}_{44}=-0.073\right),{ }^{28}$ so $\left|S_{\mathrm{r}}-S_{\theta}\right|$ is independent on $\theta_{\mathrm{S}}$. Because the stress and strain were related by the elastic tensor of the material (Eqs. (6) and (7)), the principal stress is given by

$$
\begin{aligned}
\left|P_{r}-P_{\theta}\right|= & \left\{\left[\left(C_{11}-C_{23}\right) \cos 2 \theta\right]^{2}\right. \\
& \left.+\left[2 C_{44} \sin 2 \theta\right]^{2}\right\}^{0.5}\left|S_{r}-S_{\theta}\right| .
\end{aligned}
$$

The refractive indices, photoelastic constants and elastic constants of $\mathrm{MgO}$ and $\mathrm{LiF}$ for the calculation are listed in Table I. ${ }^{29-32}$

\section{RESULTS AND DISCUSSION}

\section{A. Transient transmission images inside $\mathrm{MgO}$ and LiF single crystals}

First, we observed time-resolved transmission images of probe pulses to see what had happened inside $\mathrm{MgO}$ and $\mathrm{LiF}$ crystals after irradiation with a focused fs laser pulse. In this observation, transmission images were obtained by the optical setup of Fig. 2(a) without an analyzer. Figure 3(a) shows the transmission images at various time delays after photoexcitation inside a $\mathrm{MgO}$ crystal by a fs laser pulse of $2 \mu \mathrm{J}$. After the photoexcitation, a squircle-shaped stress wave was generated around the photoexcited region at the center. In our previous study, the generation of the stress wave was attributed to the sudden generation of thermal stress around the photoexcited region within the acoustic relaxation time. $^{7,20}$ The shape of the stress wave is the result of the direction-dependent velocity of the elastic wave due to the anisotropic elastic tensor of a $\mathrm{MgO}$ single crystal (Table I). The observed velocities of the stress wave were about $9.3 \mu \mathrm{m} / \mathrm{ns}$ in the $\langle 100\rangle$ and $10.1 \mu \mathrm{m} / \mathrm{ns}$ in the $\langle 110\rangle$. According to the elastic dynamics, ${ }^{33}$ the phase velocities of quasi-longitudinal elastic waves inside a $\mathrm{MgO}$ single crystal are calculated to be $9.1 \mu \mathrm{m} / \mathrm{ns}$ in the $\langle 100\rangle$ and $9.9 \mu \mathrm{m} / \mathrm{ns}$ in the $\langle 110\rangle$ (Table II). The theoretical velocities were close to the observed ones.

Inside a LiF single crystal (Fig. 3(b)), the propagation of cracks in the $\langle 100\rangle$ directions as well as squircle-shaped stress wave were observed at the same time. The cracks were always inside the stress wave, and the stress wave propagated more slowly than in a $\mathrm{MgO}$ single crystal. The propagation velocities of the stress wave were about $6.9 \mu \mathrm{m} / \mathrm{ns}$ in the $\langle 100\rangle$ and $7.6 \mu \mathrm{m} / \mathrm{ns}$ in the $\langle 110\rangle$, which were similar to the phase velocities of the quasi-longitudinal elastic waves calculated based on the elastic dynamics $(6.5 \mu \mathrm{m} / \mathrm{ns}$ and $7.3 \mu \mathrm{m} / \mathrm{ns}$, respectively; Table II).

The time-resolved observations suggest that the characteristic shapes of the stress waves had concentrated shear 
(a) $\mathrm{MgO}$
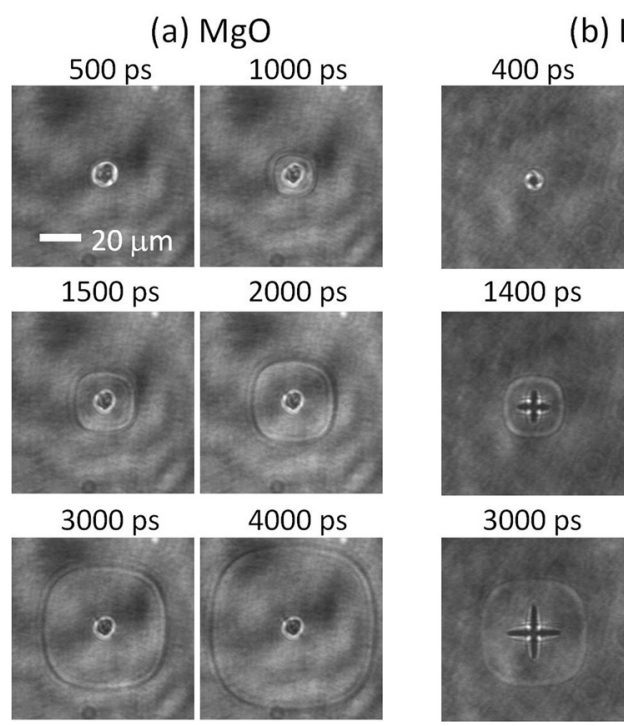

$1400 \mathrm{ps}$

2000 ps
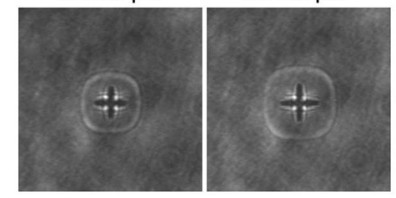

3000 ps 4000 ps

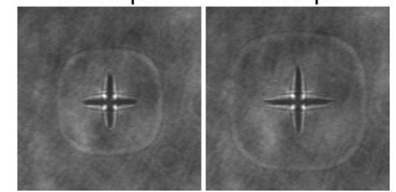

FIG. 3. Time-resolved transmission images after photoexcitation by a fs laser pulse focused inside a (a) $\mathrm{MgO}$ and (b) $\mathrm{LiF}$ single crystal. The scale bar and the orientation of the crystal were the same in (a) and (b).

stress or tensile stress in the specific regions and they could be the driving forces of the generation of the dislocation bands and cracks. However, the transmission images cannot give us any knowledge on the directions or relative amplitude of the stress and strain. Therefore, the observation of transient stress distributions is necessary for elucidating the mechanism of dislocation and crack generation.

\section{B. Strain dynamics in MgO}

\section{Transient birefringence images inside $\mathrm{MgO}$}

Figures 4(a) and 4(b) show the distributions of transient birefringence inside $\mathrm{MgO}$ after photoexcitation by a focused fs laser pulse of $1 \mu \mathrm{J}$. Two propagating birefringent regions were observed; one is a squircle-shaped stress wave, which had been observed also in the transmission image (Fig. 3(a)), and the other propagated more slowly than the squircleshaped stress wave, which could not be observed clearly in the transmission image. In our previous study, both these propagating regions were attributed to stress waves, and we called them "primary stress wave" and "secondary stress wave," respectively. The secondary stress waves propagated mainly in the $\langle 100\rangle$ direction and the velocity was about $6.4 \mu \mathrm{m} / \mathrm{ns}$, which was close to the velocity in $\langle 100\rangle$ of the quasi-transversal elastic wave in $\mathrm{MgO}\left(v^{(t)}{ }_{\langle 100\rangle}=6.6 \mu \mathrm{m} / \mathrm{ns}\right.$

TABLE II. Phase velocities of elastic waves $\left(v / \mu \mathrm{m} \mathrm{ns}^{-1}\right)$ inside $\mathrm{MgO}$ and $\mathrm{LiF}$ crystals calculated based on the elastic dynamics. $v^{(l)}{ }_{\langle 100\rangle}$ and $v^{(l)}{ }_{\langle 110\rangle}$ are the velocities of quasi-longitudinal elastic waves in the $\langle 100\rangle$ and $\langle 110\rangle$, respectively, and $v^{(t)}\langle 100\rangle$ is that of a quasi-transverse elastic wave in the $\langle 100\rangle$. They were calculated by the theoretically derived equations: $v^{(l)}\langle 100\rangle=\sqrt{C_{11} / \rho}$, $v^{(l)}{ }_{\langle 110\rangle}=\sqrt{\left(C_{11}+C_{23}+2 C_{44}\right) /(2 \rho)}, \quad v^{(t)}{ }_{\langle 100\rangle}=\sqrt{C_{44} / \rho}$ based on the elastic dynamics. ${ }^{33}$

\begin{tabular}{lccc}
\hline \hline & $v^{(l)}\langle 100\rangle$ & $v^{(l)}{ }_{\langle 110\rangle}$ & $v^{(t)}{ }_{\langle 100\rangle}$ \\
\hline $\mathrm{MgO}$ & 9.1 & 9.9 & 6.6 \\
$\mathrm{LiF}$ & 6.5 & 7.3 & 4.9 \\
\hline \hline
\end{tabular}

(a)
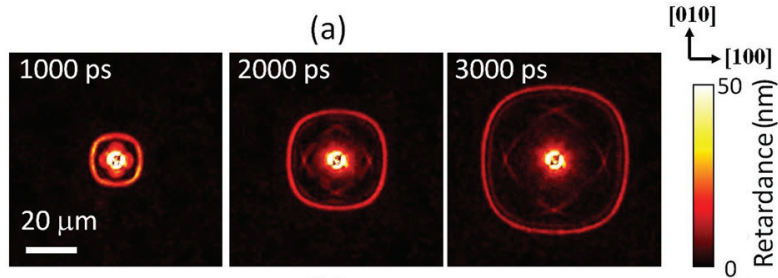

(b)
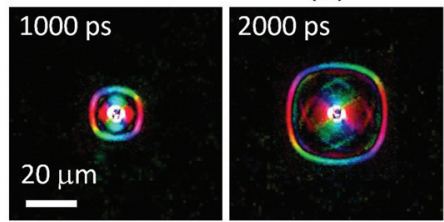

(c)
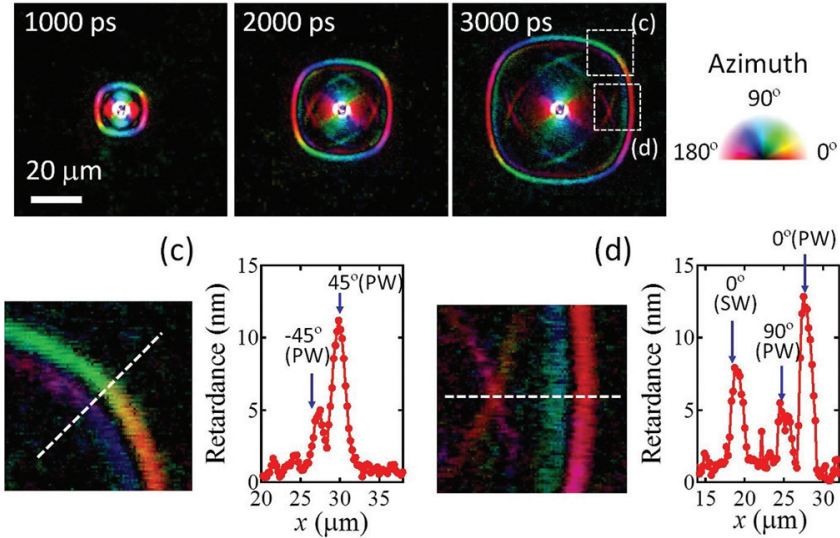

(d)

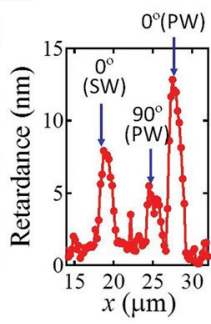

(e)
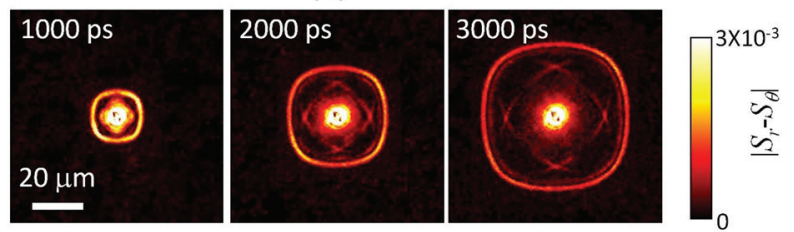

FIG. 4. (a) Retardance and (b) slow-axis azimuth distributions of transient birefringence at various time delays after photoexcitation by a fs laser pulse focused inside a $\mathrm{MgO}$ single crystal. The pulse energy was $1 \mu \mathrm{J}$. The colored semicircle indicates the relation between color and azimuth. (c) and (d) Slow-axis azimuth distributions at 3000 ps and plots of retardance along $\langle 110\rangle$ and $\langle 100\rangle$ lines (broken lines in the azimuth images), respectively. "PW" and "SW" mean "primary stress wave" and "secondary stress wave," respectively. The angles written in the plots indicate the azimuth at the retardance peaks. (e) Transient strain distributions obtained by the transient distributions of birefringence in (a) and (b).

in Table II). On the other hand, the observed velocity of the primary stress wave was similar to that of the quasilongitudinal elastic wave in $\mathrm{MgO}$ as described in Subsection III A. Because the displacements in quasi-longitudinal and quasi-transversal elastic waves are mainly parallel and perpendicular to the propagation directions, respectively, the displacement in the primary wave should be mainly parallel to the propagation direction, while that in the secondary wave should be mainly perpendicular to the propagation direction.

Figures 4(c) and 4(d) show the distributions of the slowaxis of birefringence at $3000 \mathrm{ps}$ and the plots of the retardance along $\langle 110\rangle$ and $\langle 100\rangle$ lines (broken lines in Figs. 4(c) and $4(\mathrm{~d})$, respectively). Along the $\langle 110\rangle$ line (Fig. 4(c)), the slow-axis in the front part of the primary stress wave was parallel to the propagation direction $\left(=45^{\circ}\right)$, while that in the back part was perpendicular to the propagation direction $\left(=-45^{\circ}\right)$. Also along the $\langle 100\rangle$ line (Fig. 4(d)), the slowaxes in the front part and back part of the primary stress wave were, respectively, parallel $\left(=0^{\circ}\right)$ and perpendicular $\left(=90^{\circ}\right)$ to the propagation direction. Because the primary stress wave had been attributed to a quasi-longitudinal wave, 
of which displacement should be mainly parallel to the propagation direction, the observed slow-axis distribution in the primary stress wave means that the front part of the stress wave was compressed in the propagation direction, whereas the back part was expanded in the propagation direction. On the other hand, the slow-axis in the secondary stress waves was parallel to the propagation direction $\left(0^{\circ}\right.$ along the $\langle 100\rangle$ line) (Fig. 4(d)). Because the secondary stress wave had been attributed to a quasi-transverse wave, of which displacement should be mainly perpendicular to the propagation direction, the slow-axis in the secondary stress wave means that the crystal in the secondary stress wave should have been stretched perpendicular to the propagation direction.

The birefringence around the photoexcited region was much larger than those in any other region and did not change in the observation time range. The quasi-static birefringence around the photoexcited region should be due to the thermal stress, because the thermal stress is much larger than those in stress waves as shown by an elastic simulation shown in Subsection III B 2 and the thermal diffusion would have occurred in a longer time range (after $100 \mathrm{~ns}$ ). ${ }^{13,34}$

Under the assumption that the origin of the birefringence should be anisotropic strain in a solid, the distribution of the difference between the principal strains $\left(\left|S_{r}-S_{\theta}\right|\right)$ can be obtained using the relation between strain and birefringence (Eq. (12)). The calculated distributions of $\left|S_{r}-S_{\theta}\right|$ from Figs. 4(a) and 4(b) were shown in Fig. 4(e). In the calculation, we used the material properties in Table I and $l=18$ $\mu \mathrm{m}$, which was the observed length of the structural change in the laser propagation axis. The $\left|S_{r}-S_{\theta}\right|$ distributions in the primary stress wave had maximum around the $\langle 110\rangle$ directions (Fig. 4(e)), whereas the observed retardance in the primary stress wave was almost homogeneous (Fig. 4(a)). This difference comes from the anisotropy of the photoelastic tensor of a $\mathrm{MgO}$ crystal, i.e., the strain-induced birefringence is larger in the $\langle 100\rangle$ direction than in the $\langle 110\rangle$ direction $\left(\left|\mathrm{p}_{11}-\mathrm{p}_{12}\right|>\left|2 \mathrm{p}_{44}\right|\right)$. The temporal evolution of $\left|S_{r}-S_{\theta}\right|$ in the stress wave will be described in detail in Subsection III B 3.

\section{Simulation of the transient strain distributions inside $\mathrm{MgO}$}

The thermo-elastic simulation helps us to understand deformation in the fs laser-induced stress wave. The $\left|S_{r}-S_{\theta}\right|$ distributions at 1000, 2000, and 3000 ps were shown in Fig. 5(a) to compare with the observed distributions of birefringence (Fig. 4(e)). The parameters for the simulation were $\Delta \mathrm{T}=100 \mathrm{~K}, w_{\mathrm{th}}=1 \mu \mathrm{m}$, and $l_{\mathrm{z}}=20 \mu \mathrm{m}$, which generates a stress wave of the same width as the observed ones. The $\left|S_{r}-S_{\theta}\right|$ distributions by the simulation, the shape of the stress waves, and the range of the thermal stressed region were almost the same as those by the observation. The direction of the principal strain at 3000 ps shown in Fig. 5(b) was almost same as the observed slow-axis distribution of birefringence in Fig. 4(b). Figures 5(c) and 5(d) show the plot of $\left|S_{r}-S_{\theta}\right|$ along $\langle 110\rangle$ and $\langle 100\rangle$ lines at $3000 \mathrm{ps}$ (the lines are drawn in Fig. 5(a)). The $\left|S_{r}-S_{\theta}\right|$ distributions along $\langle 110\rangle$ and $\langle 100\rangle$ lines are similar to the observed retardance distributions shown in Figs. 4(c) and 4(d). Figures 5(e) and 5(f) shows the (a) $\Delta \mathrm{T}=100 \mathrm{~K}$

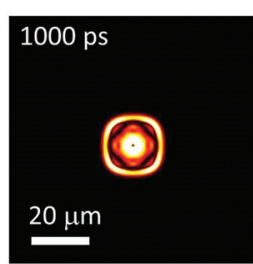

(b)

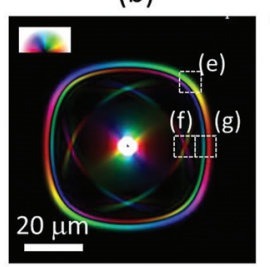

(e)

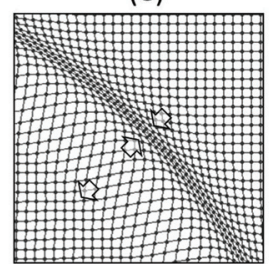

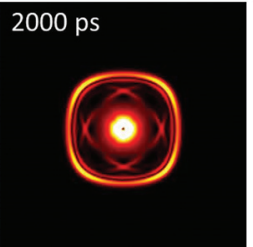

(c)

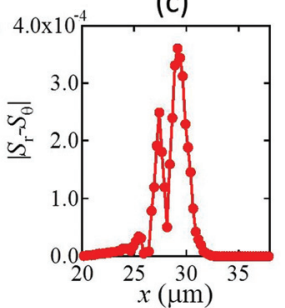

(f)

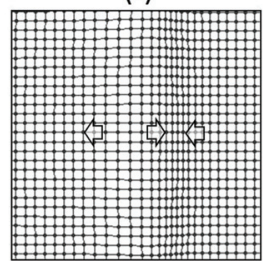

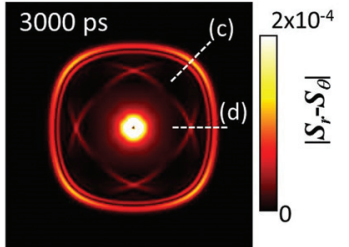

(d)

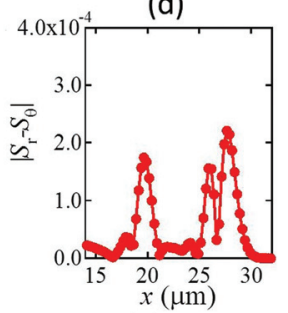

(g)
FIG. 5. (a) $\left|S_{r}-S_{\theta}\right|$ distributions at various times inside $\mathrm{MgO}$ calculated based on thermoelastic simulation. $\Delta T_{\max }=100 \mathrm{~K}, w_{\mathrm{th}}=1.0 \mu \mathrm{m}$, and $l_{\mathrm{z}}=18 \mu \mathrm{m}$. (b) Distribution of the direction of the principal strains at 3000 ps, in which the direction is represented by color. The relation between the direction and color is shown by the colored semicircle. (c) and (d) Plots of the $\left|S_{r}-S_{\theta}\right|$ at 3000 ps along $\langle 110\rangle$ and $\langle 100\rangle$ lines drawn in (a), respectively. (e)-(g) Lattice deformations at $3000 \mathrm{ps}$ in the areas shown in (b).

simulated lattice deformations at $3000 \mathrm{ps}$ around the primary stress wave. These lattice deformations show that the front part in the primary stress wave was compressed in the propagation direction, while the latter part was expanded in the propagation direction. On the other hand, in the simulated lattice deformation around the secondary stress wave shown in Fig. 5(g), the expansion perpendicular to the propagation direction predominates in the secondary stress wave. These deformations are consistent to the assignments of the primary and secondary stress waves to quasi-longitudinal and quasi-transverse waves, respectively.

\section{Amplitude of principal strain and stress in $\mathrm{MgO}$}

The amplitudes of $\left|S_{r}-S_{\theta}\right|$ (difference of principal strains) and $\left|P_{r}-P_{\theta}\right|$ (difference of principal stresses) in stress waves were calculated from the observed birefringence using Eqs. (12) and (13) and the observed length of the modified region in the beam propagation direction; $l=18 \mu \mathrm{m}$ for $1 \mu \mathrm{J}$ and $l=24 \mu \mathrm{m}$ for $2 \mu \mathrm{J}$. Here, we focused the $\left|S_{r}-S_{\theta}\right|$ and $\left|P_{r}-P_{\theta}\right|$ at the peaks in the primary and secondary stress waves along the $\langle 100\rangle$ and $\langle 110\rangle$ lines. They were denoted by $\left|\Delta S^{(\Gamma)}{ }_{\alpha}\right|$ and $\left|\Delta P^{(\Gamma)}{ }_{\alpha}\right|$ of which positions are shown in Fig. 6(a). In these notations, $\Gamma$ indicates the mode of the stress waves (i.e., $\Gamma=\mathrm{p}$ and $\Gamma=\mathrm{s}$ for primary and secondary stress waves, respectively) and $\alpha$ indicates the direction of the stressed region from the photoexcited region (i.e., $\alpha=\langle 100\rangle$ means the stress on the $\langle 100\rangle$ line.). The temporal evolutions of the $\left|\Delta S^{(\Gamma)}{ }_{\alpha}\right|$ and 
(a)

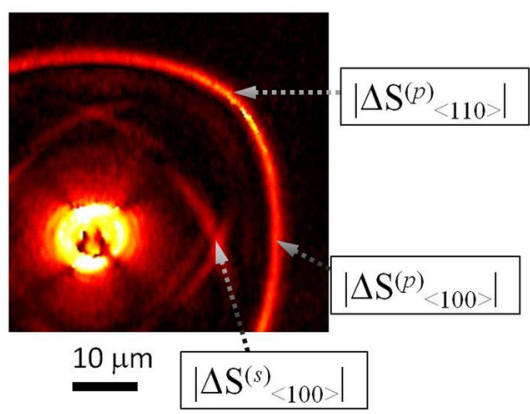

(c)

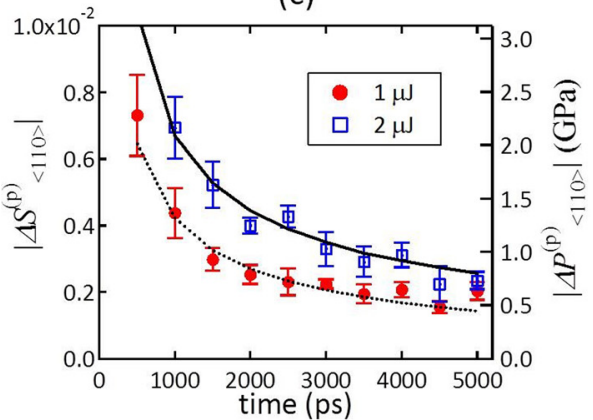

(b)

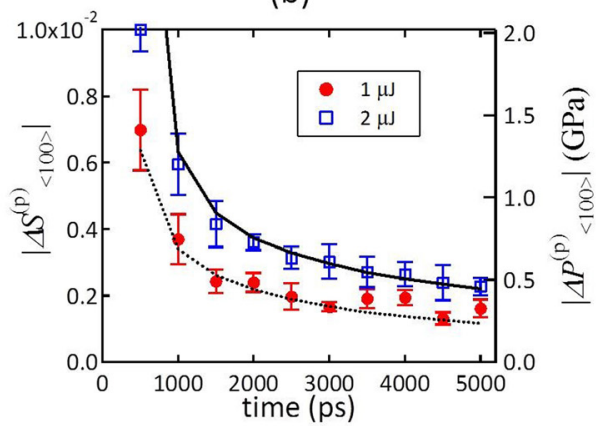

(d)

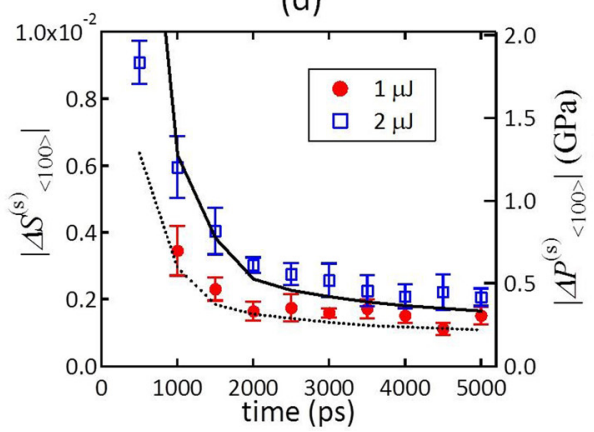

FIG. 6. (a) The positions of $\left|\Delta S^{(p)}{ }_{\langle 110\rangle}\right|$, $\left|\Delta S^{(p)}{ }_{\langle 100\rangle}\right|$, and $\left|\Delta S^{(s)}{ }_{\langle 100\rangle}\right|$. (b)-(d) The differences of principal strains and stresses inside $\mathrm{MgO}$ plotted against the time delays. Solid line and dotted line in (b) and (d) are $\left|S_{r}-S_{\theta}\right|$ by the simulation of $\Delta T_{\max }=2700 \mathrm{~K}, w_{\text {th }}=2 \mu \mathrm{m}$ and that of $\Delta T_{\max }=2300 \mathrm{~K}$ and $w_{\text {th }}$ $=1.1 \mu \mathrm{m}$, respectively, whereas those in (c) are $\left|S_{r}-S_{\theta}\right|$ by the simulation of $\Delta T_{\max }=1900 \mathrm{~K}$ and that of $\Delta T_{\max }$ $=1700 \mathrm{~K}$, respectively.
$\left|\Delta P^{(\Gamma)}{ }_{\alpha}\right|$ at the peak in the stress waves were shown in Figs. 6(b) and 6(c). The strain and stress amplitudes were in the ranges of $0.1 \times 10^{-2}-1 \times 10^{-2}$ and $0.25-2.5 \mathrm{GPa}$, respectively, and those at pulse energy of $2 \mu \mathrm{J}$ were about twice larger than those of $1 \mu \mathrm{J}$. The stress range on the $\langle 110\rangle$ line was about 1.5 times larger than those on $\langle 100\rangle$, which is because of anisotropic stress tensor in $\mathrm{MgO}$; according to Eq. (13), $\left|\Delta P_{\langle 110\rangle}\right|=2 * \mathrm{C}_{44} *\left|\Delta S_{\langle 110\rangle}\right|=312 \quad(\mathrm{GPa})^{*} \quad\left|\Delta S_{\langle 110\rangle}\right|$, while $\left|\Delta P_{\langle 100\rangle}\right|=\left(\mathrm{C}_{11}-\mathrm{C}_{23}\right)^{*}\left|\Delta S_{\langle 100\rangle}\right|=202(\mathrm{GPa})^{*}\left|\Delta S_{\langle 100\rangle}\right|$.

We found that there were small differences in the relative amplitudes of $\left|S_{r}-S_{\theta}\right|$ between the observation and simulation. To compare the differences, the ratios of $\left|\Delta S^{(p)}{ }_{\langle 110\rangle}\right|$ and $\left|\Delta S^{(s)}{ }_{\langle 100\rangle}\right|$ to $\left|\Delta S^{(p)}{ }_{\langle 100\rangle}\right\rangle$ were listed in Table III. The average ratios of $\left|\Delta S^{(s)}{ }_{\langle 100\rangle}\right|$ to $\left|\Delta S^{(p)}{ }_{\langle 100\rangle}\right|$ in $2000-6000$ ps were about 0.84 in the experiment, which were similar to that in the simulation. On the other hand, The average ratios of $\left|\Delta S^{(p)}{ }_{\langle 110\rangle}\right|$ to $\left|\Delta S^{(p)}{ }_{\langle 100\rangle}\right|$ in $2000-6000$ ps were 1.23 at $1 \mu \mathrm{J}$ and 1.34 at $2 \mu \mathrm{J}$, which were $20 \%-25 \%$ smaller than that in the simulation $\left(\left|\Delta S^{(p)}{ }_{\langle 110\rangle}\right| /\left|\Delta S^{(p)}{ }_{\langle 100\rangle}\right| \sim 1.65\right)$. These differences may come from the generation of dislocations in the $\langle 110\rangle$ directions, because the dislocation bands have been observed in $\langle 110\rangle$ after fs laser irradiation in $\mathrm{MgO}$ (Ref. 5) and it is possible that the energy of the stress wave in $\langle 110\rangle$ might have lost by generation of dislocations.

TABLE III. Ratios of $\left|\Delta \mathrm{S}^{(\mathrm{s})}{ }_{\langle 100\rangle}\right\rangle$ and $\left|\mathrm{S}^{(\mathrm{p})}{ }_{\langle 110\rangle}\right|$ to $\left|\mathrm{S}^{(\mathrm{p})}{ }_{\langle 100\rangle}\right|$ inside $\mathrm{MgO}$ by the observation and elastic simulation.

\begin{tabular}{lccccc}
\hline \hline & $\left|\Delta \mathrm{S}^{(\mathrm{s})}{ }_{\langle 100\rangle}\right\rangle /\left|\mathrm{S}^{(\mathrm{p})}{ }_{\langle 100\rangle}\right|$ & & & $\left|\Delta \mathrm{S}^{(\mathrm{p})}{ }_{\langle 110\rangle}\right| /\left|\Delta \mathrm{S}^{(\mathrm{p})}{ }_{\langle 100\rangle}\right|$ \\
\cline { 2 - 3 } \cline { 5 - 6 } & $1 \mu \mathrm{J}$ & $2 \mu \mathrm{J}$ & & $1 \mu \mathrm{J}$ & $2 \mu \mathrm{J}$ \\
\hline Observation & 0.84 & 0.84 & & 1.23 & 1.34 \\
Simulation & $( \pm 0.06)$ & $( \pm 0.05)$ & & $( \pm 0.10)$ & \multicolumn{2}{c}{$\begin{array}{c}( \pm 0.20) \\
\end{array}$} & 0.85 & 0.77 & & \multicolumn{2}{c}{1.65} \\
\hline \hline
\end{tabular}

These differences affect the estimation of the initial temperature change in the photoexcited region $\left(\Delta T_{\max }\right)$ from the comparison of the evaluated strains with those by the simulation. When the temporal evolutions of $\left|\Delta S^{(p)}{ }_{\langle 100\rangle}\right|$ and $\left|\Delta S^{(s)}{ }_{\langle 100\rangle}\right|$ by the experiment were fitted by the simulated strains, the initial temperature changes to fit the experimental values were $\Delta T_{\max }=2700 \mathrm{~K}$ for $2 \mu \mathrm{J}$ and $\Delta T_{\max }=2300 \mathrm{~K}$ for $1 \mu \mathrm{J}$ (solid and dotted lines in Figs. 6(b) and 6(d)). On the other hand, the temperature increases for the simulation to fit the temporal evolution of $\left|\Delta S^{(p)}{ }_{\langle 110\rangle}\right|$ were $\Delta T_{\max }=1900 \mathrm{~K}$ for $2 \mu \mathrm{J}$ and $\Delta T_{\max }=1700 \mathrm{~K}$ for $1 \mu \mathrm{J}$ (solid and dotted lines in Fig. 6(c)). Using these temperature changes $\left(\Delta T_{\max }=2700 \mathrm{~K}\right.$ and $2300 \mathrm{~K}$ ) and Eq. (9), the stresses at the photoexcited region are estimated to be $14 \mathrm{GPa}$ for $2 \mu \mathrm{J}$ and $12 \mathrm{GPa}$ for $1 \mu \mathrm{J}$.

\section{Strain dynamics in LiF}

\section{Transient birefringence images inside LiF}

Figures 7(a) and 7(b) show the distributions of transient birefringence inside $\mathrm{LiF}$ after photoexcitation by a focused $\mathrm{fs}$ laser pulse of $1 \mu \mathrm{J}$. A squircle-shaped birefringent wave (primary stress wave) propagated as observed in the transmission images (Fig. 3(b)). The distribution of birefringence inside the primary stress wave was completely different from that in $\mathrm{MgO}$. Whereas the birefringent regions due to the secondary stress waves in $\mathrm{MgO}$ were separated from the primary stress wave (Figs. 4(a) and 4(b)), the birefringent region inside the primary stress wave in $\mathrm{LiF}$ was distributed from the cracks to the $\langle 110\rangle$ regions in the primary stress wave (Figs. 7(a) and 7(b)). The peaks in the birefringent region inside the primary stress wave were located at the tips of cracks until the crack propagation stopped. After the crack propagation stopped (6000 ps in Fig. 7(b)), the birefringent regions inside the primary stress wave propagated away from the cracks at about $4.2 \mu \mathrm{m} / \mathrm{ns}$ in the $\langle 100\rangle$ direction. This propagation velocity is 

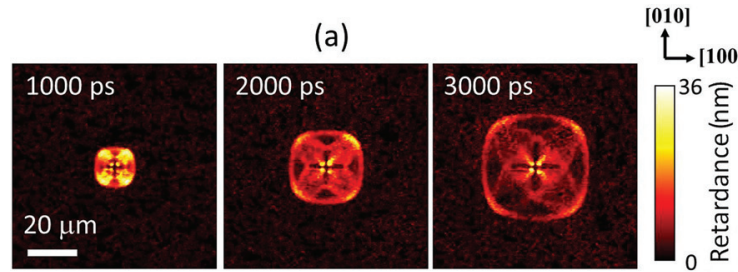

(b)
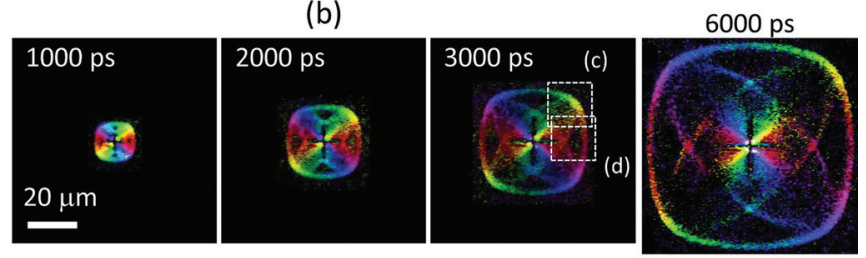

(c)
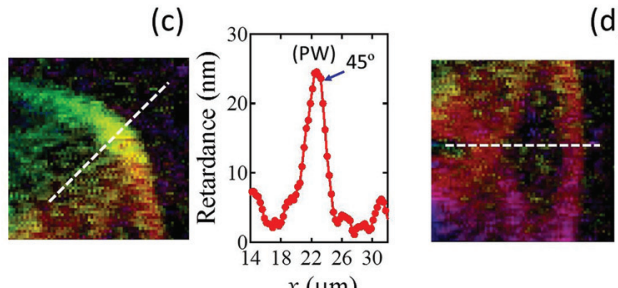

(d)

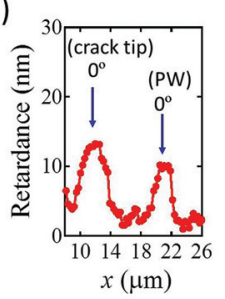

(e)
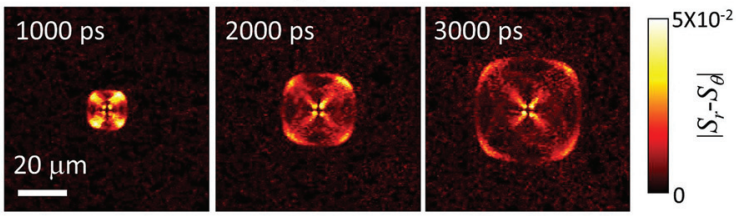

FIG. 7. (a) Retardance and (b) slow-axis azimuth distributions of transient birefringence inside a LiF single crystal at various time delays after photoexcitation by a focused fs laser pulse. The relation between color in (b) and azimuth is the same as that in Fig. 4. (c) and (d) Slow-axis azimuth distributions and plots of retardance along $\langle 110\rangle$ and $\langle 100\rangle$ lines, respectively. PW in the plot means "primary stress wave." (e) Transient strain distributions obtained from the transient birefringence images in (a) and (b).

about $85 \%$ of the phase velocity of quasi-transverse elastic wave, $4.9 \mu \mathrm{m} / \mathrm{ns}\left(v^{(t)}{ }_{\langle 100\rangle}\right.$ in Table II). Because the shape of the birefringent region at 6000 ps was similar to the strained region in the quasi-transverse elastic wave in $\mathrm{LiF}$ as described in Subsection III C 2, the birefringent peak near the crack tip should be attributed to the quasi-transverse elastic wave.

Figures 7(c) and 7(d) show the slow-axis azimuth distributions at $3000 \mathrm{ps}$ and the plots of the retardance along $\langle 110\rangle$ and $\langle 100\rangle$ lines, respectively. As observed in $\mathrm{MgO}$, the slow-axis in the primary stress wave (PW) was parallel to the propagation direction $\left(0^{\circ}\right.$ in $\langle 100\rangle$ and $45^{\circ}$ in $\left.\langle 110\rangle\right)$. Therefore, the dominant deformation in the primary stress wave in $\mathrm{LiF}$ was compression in the propagation direction. However, birefringence was not observed in the back part of the stress wave, although it was observed clearly in $\mathrm{MgO}$ (Figs. 4(c) and 4(d)). The absence of the strain in the back part of the stress wave in LiF might be due to the crack generation.

The slow axis at the birefringence peak near the crack tip was also parallel to the propagation direction. Because the birefringence peak was attributed to the quasi-transverse elastic wave, the displacement perpendicular to the propagation direction should be dominant near the crack tip. According to the slow axis parallel to the propagation direction, the expansion perpendicular to the crack should have occurred near the crack tip as predicted by the elastic simulation in $\mathrm{MgO}$ (Fig. 5(g)). This displacement direction suggests that the crack propagation in $\mathrm{LiF}$ should be mode I, separation of the crack walls under tensile stresses. ${ }^{8}$

Figure 7(e) shows the distributions of difference between principal strains $\left(\left|S_{r}-S_{\theta}\right|\right)$ calculated by birefringence distribution, Eq. (12), photoelastic constants of LiF (Table I) and observed length of the modified region in the beam propagation direction; $l=15 \mu \mathrm{m}$ for $1 \mu \mathrm{J}$ and $l=20 \mu \mathrm{m}$ for $2 \mu \mathrm{J}$. Because the ratio of $\left|\mathrm{p}_{11}-\mathrm{p}_{23}\right|$ to $\left|2 \mathrm{p}_{44}\right|$ is about 1.5 in $\mathrm{LiF}$, the principal strain in $\langle 110\rangle$ becomes larger than that in $\langle 100\rangle$ compared with the birefringence distribution (Fig. 7(a)). The distributions of $\left|S_{r}-S_{\theta}\right|$ show that the strain was concentrated in the $\langle 110\rangle$ direction and a strained region of crossed pattern appeared in the $\langle 110\rangle$ directions from the photoexcited region. The $\left|S_{r}-S_{\theta}\right|$ around the photoexcited region was much smaller than that in $\mathrm{MgO}$. The small $\left|S_{r}-S_{\theta}\right|$ suggests that the thermal stress in the photoexcited region had been relaxed due to crack generation.

\section{Simulation of the transient strain distributions inside LiF}

The $\left|S_{r}-S_{\theta}\right|$ distributions were simulated by elastic dynamics and shown in Fig. 8 (amplitude in 8(a) and direction in $8(\mathrm{~b})$ ). The parameters for the simulation were $\Delta \mathrm{T}$ $=100 \mathrm{~K}, w_{\mathrm{th}}=2.0 \mu \mathrm{m}$, and $l_{\mathrm{z}}=20 \mu \mathrm{m}$. While the observed birefringence distribution at 6000 ps (Fig. 7(b)), at which the crack propagation had stopped, is similar to the $\left|S_{r}-S_{\theta}\right|$ distribution by the simulation (6000 ps in Fig. 8(b)), there are several apparent differences in $\left|S_{r}-S_{\theta}\right|$ between the observation (Figs. 7(a) and 7(b)) and simulation (Figs. 8(a) and 8(b)) until the crack propagation stops. The first difference is the strain distribution inside the primary stress wave. In the observation, the strain was distributed between the cracks and the $\langle 110\rangle$ regions of the primary stress wave, whereas the strains inside the primary stress wave were distributed around the $\langle 100\rangle$ lines as a secondary stress wave in the simulation. The second difference is the strained region of a cross pattern in the observation (Fig. 7(d)), which did not appear in the simulation. The direction of the principal strain in the cross pattern was parallel to the $\langle 110\rangle$ lines, which means that the crystal had been compressed in the $\langle 110\rangle$ directions in the strain region of a cross pattern. Therefore, the strained region of the cross pattern might be attributed to dislocations, which can be generated by compression in the $\langle 110\rangle$ in rock-salt single crystals (Fig. 1(d)) and cannot be explained by linear elastic dynamics. The third difference is the range of strain around the photoexcited region. The strained region around the photoexcited region in the observation was much smaller than that in the simulation. Because the strain around the photoexcited region in the simulation is due to the thermal stress, the small strained region in the observation suggests that the thermal stress had been relaxed by crack generation. The fourth difference is the relative amplitudes of $\left|S_{r}-S_{\theta}\right|$. The differences between the observation and simulation were listed in Table IV. In the observation, the $\left|S_{r}-S_{\theta}\right|$ in the stress wave in $\langle 110\rangle$ 


\section{(a) $\Delta T=100 \mathrm{~K}$}
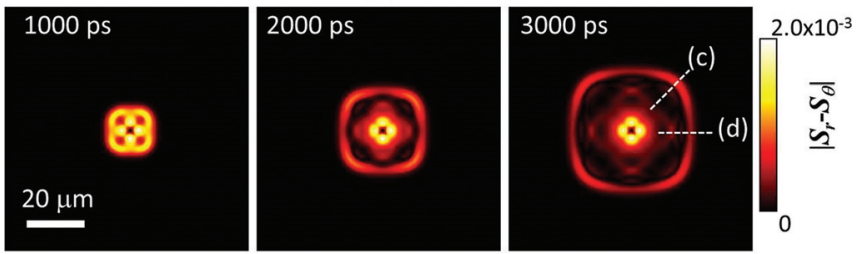

(b)
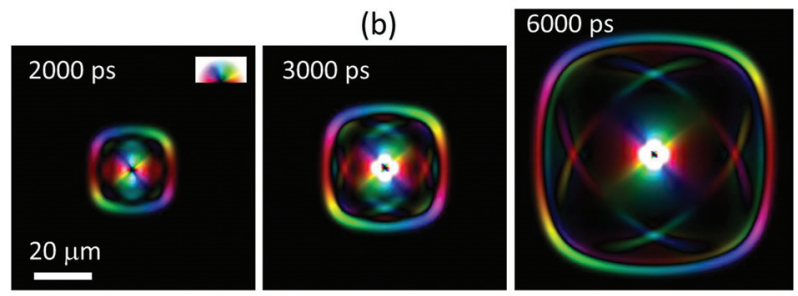

(c)

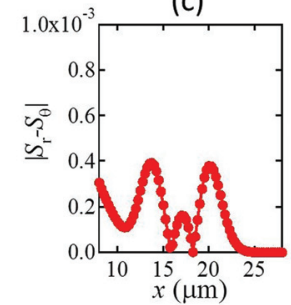

(d)

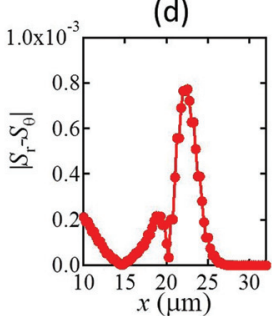

FIG. 8. (a) $\left|S_{r}-S_{\theta}\right|$ distributions at various times inside $\mathrm{LiF}$ calculated by thermoelastic simulation of $\Delta T_{\max }=100 \mathrm{~K}, w_{\mathrm{th}}=2.0 \mu \mathrm{m}$, and $l_{\mathrm{z}}=20 \mu \mathrm{m}$. (b) Distributions of the direction of the principal strains at $2000 \mathrm{ps}, 3000 \mathrm{ps}$, and $6000 \mathrm{ps}$. The relation between the direction and color is shown by the colored semicircle. (c) and (d) Plots of the principal strain at 3000 ps along $\langle 110\rangle$ and $\langle 100\rangle$, respectively.

$\left(\left|\Delta S^{(p)}\langle 110\rangle\right|\right)$ was about 2.5 times larger than that in $\langle 100\rangle$ $\left(\left|\Delta S^{(p)}{ }_{\langle 100\rangle}\right|\right)$, whereas $\left|\Delta S^{(p)}{ }_{\langle 110\rangle}\right|$ was about 2.0 times larger than $\left|\Delta S^{(p)}{ }_{\langle 100\rangle}\right|$ (Figs. 8(c) and $8(\mathrm{~d})$ ) in the simulation. In addition, the strain peak at the crack tip $\left(\left|\Delta S^{(s)}{ }_{\langle 100\rangle}\right|\right)$ was 1.3 times larger than $\left|\Delta S^{(p)}{ }_{\langle 100\rangle}\right|$ in the observation (Fig. 7(d)), whereas $\left|\Delta S^{(s)}\langle 100\rangle\right|$ was about 1.1 times larger than $\left|\Delta S^{(p)}\langle 100\rangle\right|$ in the simulation (Fig. 8(d)). These differences suggest that the strains in $\langle 110\rangle$ and at the crack tip should be enhanced by the crack formation.

\section{Amplitude of principal strain and stress in LiF}

Figure 9 shows the temporal evolution of the $\left|\Delta S^{(\Gamma)}{ }_{\alpha}\right|$ and $\left|\Delta P^{(\Gamma)}{ }_{\alpha}\right|$ at the peak in the stress waves. The strain amplitudes at the same time delays and same positions were more than 3 times larger than those in $\mathrm{MgO}$. This difference in the strain amplitudes between $\mathrm{LiF}$ and $\mathrm{MgO}$ should be due to the difference in the thermal expansion coefficients, which is $3.3 \times 10^{-5} \mathrm{~K}^{-1}$ in $\mathrm{LiF}$, whereas $1.1 \times 10^{-5} \mathrm{~K}^{-1}$ in $\mathrm{MgO}$ (Table I). In these graphs, the decay profiles of the simulated principal strains were drawn by solid and dotted lines. The simulated decay profiles (solid and dotted lines in Figs. 9(b)-9(d)) reproduced the temporal evolutions of the observed strains. By comparing the observation with the simulation in $\left|\Delta S^{(p)}{ }_{\langle 100\rangle}\right|$, we evaluated that the initial temperature change in the photoexcited region $\left(\Delta T_{\max }\right)$ should be $\Delta T_{\max }=2000 \mathrm{~K}$ at $2 \mu \mathrm{J}$ and $\Delta T_{\max }=1400 \mathrm{~K}$ at $1 \mu \mathrm{J}$ to generate the observed $\left|\Delta S^{(p)}{ }_{\langle 100\rangle}\right|$. However, these values are underestimated, because the observed strain distribution was different from the simulated one due to the crack formation. One difference between the observation and simulation is the enhancement of the strain around the $\langle 110\rangle$ lines from the photoexcited region; the observed $\left|\Delta S^{(p)}{ }_{\langle 110\rangle}\right| /\left|\Delta S^{(p)}{ }_{\langle 100\rangle}\right|$ was about 2.5, which was larger than the simulated value, 2.0 (Table IV). Therefore, when $\Delta T_{\max }$ is estimated by the comparison of $\left|\Delta S^{(p)}{ }_{\langle 110\rangle}\right|$ between the observation and simulation (solid and dotted lines in Fig. 9(c)), we obtained $\Delta T_{\max }=2500 \mathrm{~K}$ at $2 \mu$ and $\Delta T_{\max }$ $=1800 \mathrm{~K}$ at $1 \mu \mathrm{J}$, which were larger 1.25 times higher than those estimated by $\left|\Delta S^{(p)}{ }_{\langle 100\rangle}\right|$. Using these temperature changes $\left(\Delta T_{\max }=2500 \mathrm{~K}\right.$ and $\left.1800 \mathrm{~K}\right)$ and Eq. (9), the stresses at the photoexcited region are estimated to be $16 \mathrm{GPa}$ for $2 \mu \mathrm{J}$ and $11 \mathrm{GPa}$ for $1 \mu \mathrm{J}$. These stresses are comparable to those of $\mathrm{MgO}$ at the same pulse energies.

Another difference is the relative amplitudes between $\left|\Delta S^{(s)}{ }_{\langle 100\rangle}\right|$ and $\left|\Delta S^{(p)}{ }_{\langle 100\rangle}\right|$; the observed $\left|\Delta S^{(s)}{ }_{\langle 100\rangle}\right| /$ $\left|\Delta S^{(p)}{ }_{\langle 100\rangle}\right|$ was about 1.35 in average between $2000 \mathrm{ps}$ and $6000 \mathrm{ps}$, whereas the simulated one was about 1.1 in the same time range (Table IV). The difference in $\left|\Delta S^{(s)}{ }_{\langle 100\rangle}\right|$ between the observation and simulation should be due to the crack generation, because the stress tends to concentrate near the crack tip, which is known as "stress concentration." 8 Our observation showed that the stress near the crack tip was enhanced by a factor of 1.2.

\section{Comparison between MgO and LiF}

According to the time-resolved observations by a pumpprobe transmission microscope, a single stress wave was observed both in $\mathrm{MgO}$ and $\mathrm{LiF}$, and only the difference was that the crack generation observed in $\mathrm{LiF}$ and not in $\mathrm{MgO}$. On the other hand, the time-resolved birefringence imaging showed the generation of two stress waves: the faster stress wave, i.e., a primary stress wave, was attributed to a quasilongitudinal elastic wave and the slower one, a secondary stress wave, was to a quasi-transverse elastic wave. In addition, we found a lot of differences in the stress dynamics between $\mathrm{MgO}$ and $\mathrm{LiF}$ single crystals from the transient birefringence distributions. The differences between $\mathrm{MgO}$ and $\mathrm{LiF}$ were summarized as follows:

(i) The strain amplitudes in LiF (Fig. 9) were more than 3 times larger than those in $\mathrm{MgO}$ (Fig. 6) at the same pulse energy.

(ii) The strained region around the photoexcited region in $\mathrm{MgO}$ was comparable to the simulated one (Figs. 4(e) and 5(a)), whereas that in LiF was much smaller than the simulated one (Figs. 7(e) and 8(a)).

(iii) The birefringent region of the primary stress wave in $\mathrm{MgO}$ was clearly separated from that of the secondary stress wave (Figs. 4(a) and 4(b)), whereas the

TABLE IV. Ratios of $\left|\Delta \mathrm{S}^{(\mathrm{s})}{ }_{\langle 100\rangle}\right|$ and $\left|\mathrm{S}^{(\mathrm{p})}{ }_{\langle 110\rangle}\right|$ to $\left|\mathrm{S}^{(\mathrm{p})}{ }_{\langle 100\rangle}\right|$ inside LiF by the observation and elastic simulation.

\begin{tabular}{lccccc}
\hline \hline & \multicolumn{1}{|c|}{$\mathrm{S}^{(\mathrm{s})}{ }_{\langle 100\rangle}|/| \mathrm{S}^{(\mathrm{p})}{ }_{\langle 100\rangle} \mid$} & & $\left|\Delta \mathrm{S}^{(\mathrm{p})}{ }_{\langle 110\rangle}\right| /\left|\Delta \mathrm{S}^{(\mathrm{p})}{ }_{\langle 100\rangle}\right|$ \\
\cline { 2 - 3 } \cline { 5 - 6 } Observation & $1 \mu \mathrm{J}$ & $2 \mu \mathrm{J}$ & & $1 \mu \mathrm{J}$ & $2 \mu \mathrm{J}$ \\
\hline Simulation & $\begin{array}{c}1.34 \\
\pm 0.10)\end{array}$ & $\begin{array}{c}1.36 \\
( \pm 0.09)\end{array}$ & & $\begin{array}{c}2.42 \\
( \pm 0.18)\end{array}$ & $\begin{array}{c}2.50 \\
( \pm 0.20)\end{array}$ \\
\hline \hline
\end{tabular}


(a)

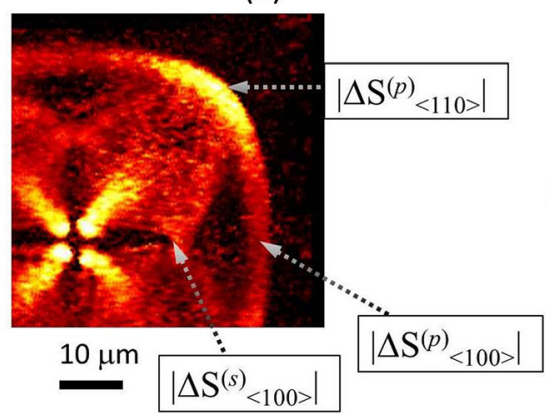

(c)

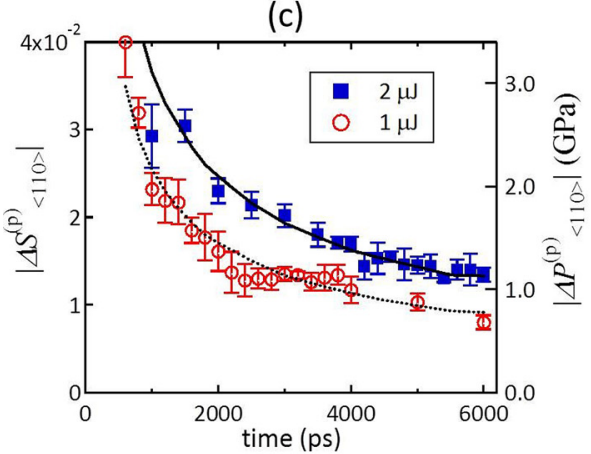

(b)

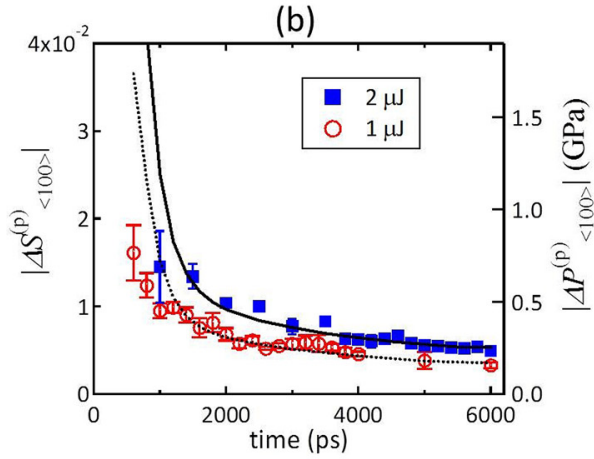

(d)

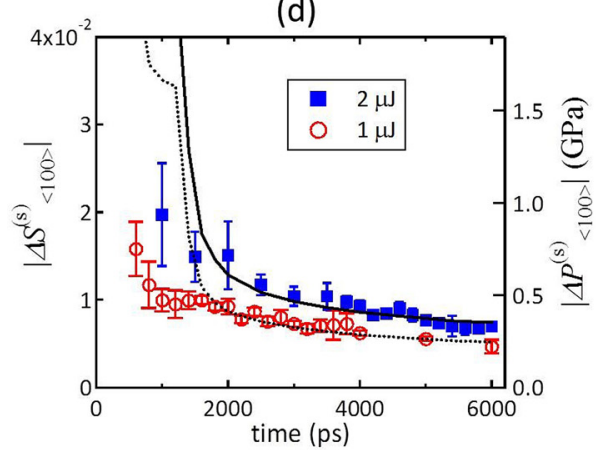

FIG. 9. (a) The positions for $\left|\Delta S^{(p)}{ }_{\langle 110\rangle}\right|,\left|\Delta S^{(p)}{ }_{\langle 100\rangle}\right|$, and $\left|\Delta S^{(s)}{ }_{100\rangle}\right|$ in LiF. (b)-(d) The differences of principal strains and stresses inside $\mathrm{LiF}$ plotted against the time delays. Solid line and dotted line in (b) are $\left|S_{r}-S_{\theta}\right|$ by the simulation of $\Delta T_{\max }$ $=1180 \mathrm{~K}, \quad w_{\text {th }}=2 \mu \mathrm{m}$ and that of $\Delta T_{\max }=880 \mathrm{~K}$ and $w_{\text {th }}=2 \mu \mathrm{m}$, respectively, those in (c) are $\left|S_{r}-S_{\theta}\right|$ by the simulation of $\Delta T_{\max }=1930 \mathrm{~K}$ and that of $\Delta T_{\max }=1320 \mathrm{~K}$, respectively, and those in (d) are $\left|S_{r}-S_{\theta}\right|$ by the simulation of $\Delta T_{\max }=2200 \mathrm{~K}$ and that of $\Delta T_{\max }=1200 \mathrm{~K}$, respectively. birefringence in LiF was distributed between the primary and secondary stress waves (Figs. 7(a) and 7(b)). The birefringent regions of the primary and secondary stress waves in LiF were separated from each other after the crack propagation stopped (6000 ps in Fig. 7(b)).

(iv) The observed ratio of $\left|\Delta S^{(p)}{ }_{\langle 110\rangle}\right|$ to $\left|\Delta S^{(p)}{ }_{\langle 100\rangle}\right|$ in $\mathrm{MgO}$ was $20 \%-25 \%$ smaller than that by the simulation (Table III), whereas that in LiF was about 1.25 times larger than that of the simulated value (Table IV).

(v) The ratio of $\left|S_{r}-S_{\theta}\right|$ in the secondary stress wave $\left(\left|\Delta S^{(s)}{ }_{\langle 100\rangle}\right|\right)$ to that in the primary stress wave in $\langle 100\rangle\left(\left|\Delta S^{(p)}{ }_{\langle 100\rangle}\right|\right)$ in $\mathrm{MgO}$ was nearly same as that of the simulation ( 0.81 both in the experiment and simulation), whereas the observed ratio in $\mathrm{LiF}$ was about 1.2 times larger than that of the simulation (Table III).

(vi) In $\mathrm{LiF}$, birefringent regions of a cross pattern appeared in the $\langle 110\rangle$ directions from the photoexcited region at several ns after photoexcitation (Fig. 7(e)), although they were not observed in the simulation (Fig. 8(a)) and in $\mathrm{MgO}$ (Fig. 4(e)).

These differences can be interpreted based on the difference in properties between $\mathrm{MgO}$ and $\mathrm{LiF}$ and crack generation, which occurred only in LiF. The difference of (i) (larger strain amplitudes in LiF at the same energy) could be due to larger thermal expansion coefficient of $\mathrm{LiF}(\beta=3.3$ $\times 10^{-5}$ ), which is about 3 times larger than that of $\mathrm{MgO}$ $\left(\beta=1.1 \times 10^{-5}\right.$; Table I). On the other hand, the other differences [(ii)-(vi)] could be originated from the crack generation only in $\mathrm{LiF}$, because these differences mean that the observed strain distributions inside $\mathrm{MgO}$ could be reproduced by a linear elastic simulation, whereas the observed strain dynamics was different from the simulated ones inside a $\mathrm{LiF}$ except the velocities of the stress waves. The difference of (ii) should be due to the relaxation of the thermal stress around the photoexcited region due to the crack generation inside LiF. The differences of (iii) and (iv) mean the enhancement of the strains in LiF. The larger strain in $\langle 110\rangle$ inside LiF suggests that the crack generation inside $\mathrm{LiF}$ created free boundaries on the $\{100\}$ planes, which could facilitate the displacement from the $\{100\}$ surface of the cracks to the $\{110\}$ planes. The difference of (v) could be due to the concentration of strains near the crack tip inside LiF. $^{8}$ The difference of (vi) suggests that the birefringent regions in the $\langle 110\rangle$ directions from the photoexcited region inside $\mathrm{LiF}$ could be originated from dislocations, because the slip plane and direction in rock-salt crystals are of $\{110\}$ and $\langle 110\rangle$, respectively, and strain field is generated around a dislocation. ${ }^{1}$ The slow axes of the birefringent regions, which were parallel to the radial directions $(\langle 110\rangle)$, indicate that compression occurred in the radial directions in the birefringent region. Therefore, the dislocations could be generated by the compression in the $\langle 110\rangle$ directions by thermal expansion in the photoexcited region and crack generation.

From the time-resolved strain observation, we found that the strain distribution became different from the simulation due to crack generation inside LiF. Then, why are cracks generated more easily by fs laser irradiation inside LiF than inside $\mathrm{MgO}$ ? First, the reason was discussed in terms of differences in the elastic energy due to thermal expansion and surface energy. Initialization of crack propagation by photoexcitation should be related to the elastic energy by thermal expansion in the photoexcited region and surface energy that must be overcome to generate cracks. ${ }^{8}$ The elastic energy density, $E_{\text {elast }}$, can be expressed roughly by

$$
E_{\text {elast }} \sim \frac{1}{2} k \varepsilon^{2}
$$

where $k$ is the elastic constant and $\varepsilon$ is the strain. According to Table I, thermal expansion coefficient of $\mathrm{LiF}$ is about 3 times larger than that of $\mathrm{MgO}$, which results in 3.0 times 
larger strain in $\mathrm{LiF}$ than in $\mathrm{MgO}\left(\varepsilon_{(\mathrm{LiF})} \sim 3 \times \varepsilon_{(\mathrm{MgO})}\right)$ under the same temperature change in a photoexcited region. Because the elastic constants of $\mathrm{LiF}$ are about 3 times smaller than those of $\mathrm{MgO}$ in average $\left(k_{(\mathrm{LiF})} \sim k_{(\mathrm{MgO})} / 3\right)$, the elastic energy due to thermal expansion in $\mathrm{LiF}$ should be 3 times larger than that of $\mathrm{MgO}$. In addition, the surface energy of $\mathrm{LiF}$ is 3.5 times smaller than that of $\mathrm{MgO}$. Therefore, comparison of elastic and surface energies suggests that larger temperature change is necessary to initiate crack generation in $\mathrm{MgO}$ than in $\mathrm{LiF}$.

As another reason that cracks are generated more easily in LiF, we focused the difference in Poisson's ratios between $\mathrm{LiF}$ and $\mathrm{MgO}$. Poisson ratio is defined as the ratio of lateral expansion to longitudinal contraction by application of compressive stress. $^{22}$ According to the paper by Dr. Ballato, ${ }^{35}$ the Poisson's ratio of a cubic crystal in the crack propagation direction $(\langle 100\rangle)$ is given by

$$
\nu_{12}=C_{23} /\left(C_{11}+C_{23}\right) .
$$

Using $C_{\mathrm{ij}} \mathrm{S}$ in Table $\mathrm{I}$, the Poisson ratio of $\mathrm{MgO}$ is $\nu_{12(\mathrm{MgO})}=0.24$, while that of $\mathrm{LiF}$ is $\nu_{12(\mathrm{LiF})}=0.27$. Because the thermal expansion in the crack propagation direction induces tensile strain perpendicular to the crack by the factor of the Poisson ratio, the difference in the Poisson's ratio between $\mathrm{LiF}$ and $\mathrm{MgO}$ means that the strain to induce crack initialization in $\mathrm{LiF}$ is slightly larger (about 1.1 times $\left.=\nu_{12(\mathrm{LiF})} / \nu_{12(\mathrm{MgO})}\right)$ than that in $\mathrm{MgO}$. Therefore, the differences in thermal expansion coefficient, surface energy, and elastic tensor between $\mathrm{LiF}$ and $\mathrm{MgO}$ should determine whether cleavage cracks are generated by fs laser irradiation.

\section{CONCLUSION}

In conclusion, we observed the strain dynamics of fs laser-induced stress wave and deformation inside $\mathrm{LiF}$ and $\mathrm{MgO}$ single crystals by a pump-probe polarization microscope. The observed strain dynamics showed that the strain distributions were different with and without generation of cracks. Inside a $\mathrm{MgO}$ single crystal, in which cracks were not generated, the observed strain dynamics could be reproduced by the simulated strain dynamics after thermal expansion in the photoexcited region, whereas inside a LiF single crystal, the strain distributions during crack propagation were completely different from the simulated ones. In particular, the strain was distributed from cracks to the $\langle 110\rangle$ regions of a stress wave and the strain in the $\langle 110\rangle$ region was enhanced. Because this study is the first time that the amplitude and distribution of strain during crack propagation in a nanosecond time-range are elucidated to our knowledge, the observed strain dynamics could be an important clue to elucidate the mechanism of shockinduced modifications inside crystals.

\section{ACKNOWLEDGMENTS}

This research was supported partially by Japan Society for the Promotion of Science (JSPS) Grant-in-Aid for
Scientific Research (C), No. 26410240 and the Crossministerial Strategic Innovation Promotion Program (SIP) of New Energy and Industrial Technology Development Organization of Japan.

${ }^{1}$ W. D. Kingery, H. K. Bowen, and D. R. Uhlmann, Introduction to Ceramics, 2nd ed. (John Wiley \& Sons, Inc., New York, 1976), Chaps. 4, 14 , and 15 .

${ }^{2}$ G. Meurant, Surface Effects in Adhesion Friction Wear and Lubrication (Elsevier, New York, 1981), p. 147.

${ }^{3}$ J. L. Robins, T. N. Rhodin, and R. L. Gerlach, J. Appl. Phys. 37, 3893 (1966).

${ }^{4}$ Z. Y. Wang, M. P. Harmer, and Y. T. Chou, Mater. Lett. 7, 224 (1988).

${ }^{5}$ S. Kanehira, K. Miura, K. Fujita, K. Hirao, J. Si, N. Shibata, and Y. Ikuhara, Appl. Phys. Lett. 90, 163110 (2007).

${ }^{6}$ C. Moon, S. Kanehira, M. Nishi, K. Miura, T. Nakaya, E. Tochigi, N. Shibata, Y. Ikuhara, and K. Hirao, J. Am. Ceram. Soc. 92, 3118 (2009).

${ }^{7}$ M. Sakakura, T. Tochio, M. Eida, Y. Shimotsuma, S. Kanehira, M. Nishi, K. Miura, and K. Hirao, Opt. Express 19, 17780 (2011).

${ }^{8}$ B. Lawn, Fracture of Brittle Solids, 2nd ed. (Cambridge University Press, New York, 1993), Chap. 2.

${ }^{9}$ J. Wong, J. L. Ferriera, E. F. Lindsey, D. L. Haupt, I. D. Hutcheon, and J. H. Kinney, J. Non-Cryst. Solids 352, 255 (2006).

${ }^{10}$ E. N. Glezer, C. B. Schaffer, N. Nishimura, and E. Mazur, Opt. Lett. 22, 1817 (1997).

${ }^{11}$ G. Paltauf and P. Dyer, Chem. Rev. 103, 487 (2003).

${ }^{12}$ M. Sakakura and M. Terazima, Phys. Rev. B 71, 024113 (2005).

${ }^{13}$ A. Vogel, J. Noack, G. Hüttman, and G. Paltauf, Appl. Phys. B 81, 1015 (2005).

${ }^{14}$ S. Juodkazis, K. Nishimura, S. Tanaka, H. Misawa, E. Gamaly, B. LutherDavies, L. Hallo, P. Nicolai, and V. Tikhonchuk, Phys. Rev. Lett. 96, 166101 (2006).

${ }^{15}$ A. Mermillod-Blondin, J. Bonse, A. Rosenfeld, I. V. Hertel, Y. P. Meshcheryakov, N. M. Bulgakova, E. Audouard, and R. Stoian, Appl. Phys. Lett. 94, 041911 (2009).

${ }^{16}$ Y. Hayasaki, M. Isaka, A. Takita, and S. Juodkazis, Opt. Express 19, 5725 (2011).

${ }^{17} \mathrm{~K}$. Matthews, Crystran Handbook of Infra-Red and Ultra-Violet Optical Materials, Electric edition (Crystran Ltd., Poole, UK, 2008).

${ }^{18} \mathrm{M}$. Born and E. Wolf, Principle of Optics, 7th ed. (Cambridge University Press, New York, 1999), Chap. 15.

${ }^{19}$ M. Yamada, Rev. Sci. Instrum. 64, 1815 (1993).

${ }^{20}$ T. Tochio, M. Sakakura, Y. Shimotsuma, M. Nishi, K. Hirao, and K. Miura, Jpn. J. Appl. Phys., Part 1 51, 126602 (2012); M. Sakakura, T. Tochio, Y. Ishiguro, M. Nakabayashi, Y. Shimotsuma, K. Hirao, and K. Miura, Proc. SPIE 8530, 853003 (2012).

${ }^{21}$ See supplementary material at http://dx.doi.org/10.1063/1.4926614 for determination of the zero delay.

${ }^{22}$ A. Yariv, Optical Electronics in Modern Communications, 5th ed. (Oxford University Press, New York, 1997), Chap. 1.

${ }^{23}$ L. D. Landau and E. M. Lifshitz, Theory of Elasticity (Pergamon, Oxford, 1986), Chaps. I-III.

${ }^{24}$ E. H. Bogardus, J. Appl. Phys. 36, 2504 (1965).

${ }^{25}$ C. V. Briscoe and C. F. Squire, Phys. Rev. 106, 1175 (1957).

${ }^{26}$ R. Ruppin, Solid State Commun. 9, 1387 (1971).

${ }^{27}$ S. Lambade, G. Sahasrabudhe, and S. Rajagopalan, J. Appl. Phys. 78, 6525 (1995).

${ }^{28}$ J. Schroeder, J. Non-Cryst. Solids 40, 549 (1980).

${ }^{29}$ K. Vedam and D. Schmidt, Phys. Rev. 146, 548 (1966).

${ }^{30}$ H. H. Li, J. Phys. Chem. Ref. Data 9, 161 (1980).

${ }^{31}$ Z. H. Levine, J. H. Burnett, and E. L. Shirley, Phys. Rev. B 68, 155120 (2003).

${ }^{32}$ J. J. Gilman, J. Appl. Phys. 31, 2208 (1960).

${ }^{33}$ M. J. Musgrave, Rep. Prog. Phys. 22, 74 (1959).

${ }^{34}$ M. Sakakura, M. Terazima, Y. Shimotsuma, K. Miura, and K. Hirao, Opt. Express 15, 16800 (2007).

${ }^{35}$ A. Ballato, IEEE Trans. Ultrason. Ferroelectr. Freq. Control 43, 56 (1996). 